\title{
Mimetic Spectral Element Method for Anisotropic Diffusion
}

\author{
Marc Gerritsma, Artur Palha, Varun Jain and Yi Zhang
}

\begin{abstract}
This paper addresses the topological structure of steady, anisotropic, inhomogeneous diffusion problems. Two discrete formulations: a) mixed and b) direct formulations are discussed. Differential operators are represented by sparse incidence matrices, while weighted mass matrices play the role of metric-dependent Hodge matrices. The resulting mixed formulations are point-wise divergence-free if the right hand side function $f=0$. The method is inf-sup stable and displays optimal convergence on orthogonal and non-affine grids.
\end{abstract}

\section{Introduction}

Anisotropic and inhomogeneous diffusion appears in many applications such as heat transfer [15], flow through porous media [87], turbulent fluid flow [116], image processing [98] or plasma physics [112]. In 2D, steady, anisotropic diffusion is governed by the following elliptic partial differential equation

$$
-\nabla \cdot(\mathbb{K} \nabla p)=f
$$

\footnotetext{
Marc Gerritsma

Faculty of Aerospace Engineering, Delft University of Technology, Kluyverweg 1, Delft, The Netherlands, e-mail:m.i.gerritsma@tudelft.nl

Artur Palha

Department of Mechanical Engineering, Eindhoven University of Technology, de Zaale, Eindhoven, The Netherlands, e-mail:a.palha@tue.nl

Varun Jain

Faculty of Aerospace Engineering, Delft University of Technology, Kluyverweg 1, Delft, The Netherlands, e-mail: v.jainetudelft.nl

Yi Zhang

Faculty of Aerospace Engineering, Delft University of Technology, Kluyverweg 1, Delft, The Netherlands, e-mail: y.zhang-14@tudelft.nl
} 
Here, $p$ is the flow potential, $f$ the source term, with $p=\bar{p}$ along $\Gamma_{p}$ and $(\mathbb{K} \nabla p, \boldsymbol{n})=$ $\bar{u}_{n}$ along $\Gamma_{u}$. Here, for all $\boldsymbol{x}, \mathbb{K}(\boldsymbol{x})$ is a symmetric, positive definite tensor.

In the presence of strong anisotropy, i.e. large ratio between the smallest and largest eigenvalues of the diffusion tensor, the construction of robust and efficient discretizations becomes particularly challenging. Under these conditions, the convergence rates of the discretization error can be considerably reduced; this effect is commonly referred in the literature as locking effect, see for example [4, 5, 12, 84]. For sufficiently refined discretizations, the deterioration of the convergence rates eventually disappears. Unfortunately, this may occur only when the grid cell size is prohibitively small.

Another important aspect is mesh flexibility. In many applications of diffusion equations, particularly in porous media flow, typical grids are highly irregular. In many of these situations the results obtained are strongly dependent on the grid type, see [11] for a discussion of the use and properties of different grids in reservoir modelling.

\subsection{Overview of standard discretizations}

In order to overcome these limitations and improve the efficiency and robustness of the discretization of the anisotropic diffusion equations, several approaches have been proposed.

The discretization of the anisotropic diffusion equations in complex media in many situations is still a trade-off between, e.g. [89]:

- Accuracy in the representation of the medium (complex grids).

- Accuracy in the discretization of the equations.

The need for such a choice is rooted in the use of numerical schemes based on two-point flux approximations (TPFA), see for example, [3, 89, 120]. These methods produce good approximations on orthogonal grids when the diffusion tensor $\mathbb{K}$ is diagonal, but are known to introduce significant discretization errors in the presence of a non-diagonal diffusion tensor. This introduces severe limitations into the possible grid choices. Under these conditions, the geometric flexibility introduced by perpendicular bisector (PEBI) grids, [11, 67, 90], is considerably limited, for example.

It has been known that the discretization error is related to the misalignment between the grid and the principal directions of the diffusion tensor $\mathbb{K}$. In fact, Aavatsmark showed in [3] that for TPFA this misalignment leads to the discretization of the wrong diffusion tensor.

These ideas initially led to the construction of grids aligned with the principal axis of the diffusion tensor, so called $\mathbb{K}$-orthogonal grids, see for example [65, 67]. This approach significantly improves the performance of the numerical method but substantially limits the geometric flexibility. 
More recently, multipoint flux-approximation (MPFA) schemes have been introduced specifically to address these limitations, see e.g. the initial works by Aavatsmark [4, 5] or a more recent presentation [2], and by Edwards and Rogers [57]. This method is based on a cell-centred finite volume formulation and introduces a dual grid in order to generate shared sub-cells and sub-interfaces. This in turn produces a discretization of the flux between two cells that involves a linear combination of several adjacent cells. This method is robust and locally conservative but does not guarantee a resulting symmetric discrete diffusion operator. More recently, this work has been connected to the mixed finite element method, [56].

Alternative approaches based on the finite element formulation have also been proposed by several authors. We briefly mention the work on the control-volume finite element discretization by Forsyth [60] and Durlofsky [54], on nodal Galerkin finite elements by Young [122], and on mixed finite elements by Durlofsky [53].

\subsection{Overview of mimetic discretizations}

Over the years, the development of numerical schemes that preserve some of the structures of the differential models they approximate has been identified as an important ingredient of numerical analysis. One of the contributions of the formalism of mimetic methods is to identify differential geometry as the proper language in which to encode these structures/symmetries. Another novel aspect of mimetic discretizations is the identification and separation of physical field laws into two sets: (i) topological relations (metric-free), and (ii) constitutive relations (metric dependent). Topological relations are intimately related to conservation laws and can (and should) be exactly represented on the computational grid. Constitutive relations include all material properties and therefore are approximate relations. For this reason, all numerical discretization error should be included in these equations. A general introduction and overview of spatial and temporal mimetic/geometric methods can be found in [39, 42, 66, 100].

The relation between differential geometry and algebraic topology in physical theories was first established by Tonti [117]. Around the same time Dodziuk [52] set up a finite difference framework for harmonic functions based on Hodge theory. Both Tonti and Dodziuk introduce differential forms and cochain spaces as the building blocks for their theory. The relation between differential forms and cochains is established by the Whitney map ( $k$-cochains $\rightarrow k$-forms) and the de Rham map ( $k$-forms $\rightarrow k$-cochains). The interpolation of cochains to differential forms on a triangular grid was already established by Whitney, [119]. These generalized interpolatory forms are now known as Whitney forms.

Hyman and Scovel [75] set up the discrete framework in terms of cochains, which are the natural building blocks of finite volume methods. Later, Bochev and Hyman [18] extended this work and derived discrete operators such as the discrete wedge product, the discrete codifferential, and the discrete inner products. 
Robidoux, Hyman, Steinberg and Shashkov, [74, 76, 78, 107, 108, 111, 113, 114] used symmetry considerations to construct discretizations on rough grids, within the finite difference/volume setting . In a more recent paper by Robidoux and Steinberg [110] a finite difference discrete vector calculus is presented. In that work, the differential operators grad, curl and div are exactly represented at the discrete level and the numerical approximations are all contained in the constitutive relations, which are already polluted by modeling and experimental error. For mimetic finite differences, see also the work of Brezzi et al. [32, 33] and Beirão da Veiga et al. [47].

The application of mimetic ideas to unstructured triangular staggered grids has been extensively studied by Perot, [99, 101-103, 123], specially in [100] where the rationale of preserving symmetries in numerical algorithms is well described. The most geometric approach is presented in the work by Desbrun et al. [49, 58, 86, 97] and the thesis by Hirani [72].

The Japanese papers by Bossavit, [26-30], serve as an excellent introduction and motivation for the use of differential forms in the description of physics and the use in numerical modeling. The field of application is electromagnetism, but these papers are sufficiently general to extend to other physical theories.

In a series of papers by Arnold, Falk and Winther, [8-10], a finite element exterior calculus framework is developed. Higher order methods are described by Rapetti [104, 105] and Hiptmair [71]. Possible extensions to spectral methods were described by Robidoux, [109]. A different approach for constructing arbitrary order mimetic finite elements has been proposed by the authors [31, 64, 92, 96], with applications to advection problems [95], Stokes' flow [81], MHD equilibrium [94], Navier-Stokes [93], and within a Least-Squares finite element formulation [16, 62, 63, 91].

Extensions of these ideas to polyhedral meshes have been proposed by Ern, Bonelle and co-authors in [22-25], by di Pietro and co-authors in [50, 51], by Brezzi and co-authors in [34], and by Beirão da Veiga and co-authors in [44-46, 48]. These approaches provide more geometrical flexibility while maintaining fundamental structure preserving properties.

Mimetic isogeometric discretizations have been introduced by Buffa et al. [40], Evans and Hughes [59], and Hiemstra et al. [70].

Another approach to develop a discretization of the physical field laws is based on a discrete variational principle for the discrete Lagrangian action. This approach has been used in the past to construct variational integrators for Lagrangian systems, e.g. [79, 85]. Kraus and Maj [80] have used the method of formal Lagrangians to derive generalized Lagrangians for non-Lagrangian systems of equations. This allows to apply variational techniques to construct structure preserving discretizations on a much wider range of systems. Recently, Bauer and Gay-Balmaz presented variational integrators for elastic and pseudo-incompressible flows [14].

Due to the inherent challenges in discretizing the diffusion equations with anisotropic diffusion tensor $\mathbb{K}$, several authors have explored different mimetic discretizations of these equations. Focussing on generalized diffusion equations we highlight [13, 69, 74, 76-78, 102, 107, 108, 111, 113, 114] for a finitedifference/finite-volume setting, [23, 36-38] for polyhedral discretizations, and 
[19, 21, 96, 106, 121] for a finite element/mixed finite element setting. For applications to Darcy flow equations and reservoir modelling see for example [1, 6, 7, 55, 73, 83, 89].

\subsection{Outline of chapter}

In Section 2 the topological structure of anisotropic diffusion problems is discussed. In Section 3 spectral basis functions are introduced which are compatible with the topological structure introduced in Section 2 . In Section 4 transformation to curvilinear elements is discussed. Results of the proposed method are presented in Section 5

\section{Anisotropic diffusion / Darcy problem}

Let $\Omega \subset \mathbb{R}^{d}$ be a contractible domain with Lipschitz continuous boundary $\partial \Omega=$ $\Gamma_{p} \cup \Gamma_{u}, \Gamma_{p} \cap \Gamma_{u}=\varnothing$. The steady anisotropic diffusion problem is given by

$$
-\nabla \cdot(\mathbb{K} \nabla p)=f,
$$

with $p=\bar{p}$ along $\Gamma_{p}$ and $(\mathbb{K} \nabla p, \boldsymbol{n})=\bar{u}_{n}$ along $\Gamma_{u}$. Here, for all $\boldsymbol{x}, \mathbb{K}(\boldsymbol{x})$ is a symmetric, positive definite tensor, i.e. there exist constants $\alpha, C>0$ such that

$$
\alpha \boldsymbol{\xi}^{T} \boldsymbol{\xi} \leq \boldsymbol{\xi}^{T} \mathbb{K}(\boldsymbol{x}) \boldsymbol{\xi} \leq C \boldsymbol{\xi}^{T} \boldsymbol{\xi} .
$$

If $\Gamma_{p} \neq \varnothing$, then (2) has a unique solution. If $\Gamma_{p}=\varnothing$ then 2 only possesses solutions if

$$
\int_{\partial \Omega} \bar{u}_{n} \mathrm{~d} S=\int_{\Omega} f \mathrm{~d} \Omega
$$

in which case the solution, $p$, is determined up to a constant.

An equivalent first order system is obtained by introducing $\boldsymbol{u}=-\mathbb{K} \nabla p$ in which case (2) can be written as

$$
\left\{\begin{array} { l l } 
{ \boldsymbol { u } + \mathbb { K } \nabla p = 0 } & { \text { in } \Omega } \\
{ \nabla \cdot \boldsymbol { u } = f } & { \text { in } \Omega }
\end{array} \text { with } \left\{\begin{array}{ll}
(\boldsymbol{u}, \boldsymbol{n})=\bar{u}_{n} & \text { along } \Gamma_{u} \\
p=\bar{p} & \text { along } \Gamma_{p}
\end{array} .\right.\right.
$$

An alternative first-order formulation is given by

$$
\left\{\begin{array} { l l } 
{ \boldsymbol { v } - \nabla p = 0 } & { \text { in } \Omega } \\
{ \boldsymbol { u } + \mathbb { K } \boldsymbol { v } = 0 } & { \text { in } \Omega } \\
{ \nabla \cdot \boldsymbol { u } = f } & { \text { in } \Omega }
\end{array} \quad \text { with } \quad \left\{\begin{array}{ll}
(\boldsymbol{u}, \boldsymbol{n})=\bar{u}_{n} & \text { along } \Gamma_{u} \\
p=\bar{p} & \text { along } \Gamma_{p}
\end{array}\right.\right.
$$


Formulation (3) is generally referred to as the Darcy problem, while the relation $\boldsymbol{u}=-\mathbb{K} \nabla p$ is called Darcy's law, [87]. The Darcy problem plays an important role in reservoir engineering. In this case $\boldsymbol{u}$ is the flow velocity in a porous medium and $p$ denotes the pressure.

While the formulations (2), (3) and (4) are equivalent, (2) only has 1 unknown, $p$, 33 has $(d+1)$ unknowns, $p$ and the $d$ components of $\boldsymbol{u}$, and (4) has $(2 d+1)$ unknowns. Formulation (4) is of special interest, because it decomposes the anisotropic diffusion problem into two topological conservation laws and one constitutive law ${ }^{1}$. By making a suitable choice where and how to represent the unknowns on a grid, the topological relations, $\boldsymbol{v}-\nabla p=0$ and $\nabla \cdot \boldsymbol{u}=f$ reduce to extremely simple algebraic relations which depend only on the topology of the mesh and are independent of the mesh size, independent of the shape of the mesh, and independent of the order of the numerical scheme. We will refer to such discretizations as exact discrete representations.

\subsection{Gradient relation}

Consider two points $A, B \in \Omega$ and a curve $\mathscr{C}$ which connects these two points, then

$$
\boldsymbol{v}-\nabla p=0 \quad \Longrightarrow \quad \overline{\boldsymbol{v}}_{\mathscr{C}}:=\int_{\mathscr{C}} \boldsymbol{v} \cdot \mathrm{d} l=\int_{A}^{B} \boldsymbol{v} \cdot \mathrm{d} l=\int_{A}^{B} \nabla p \cdot \mathrm{d} l=p(B)-p(A),
$$

where $\mathrm{d} l$ is a small increment along the curve $\mathscr{C}$.

Suppose that we take another curve $\tilde{\mathscr{C}}$ which connects the two points $A$ and $B$ then we also have

$$
\overline{\boldsymbol{v}}_{\tilde{\mathscr{C}}}:=\int_{\tilde{\mathscr{C}}} \boldsymbol{v} \cdot \mathrm{d} l=p(B)-p(A),
$$

The integral along $\mathscr{C}$ is equal to the integral along $\tilde{\mathscr{C}}$. We will refer to $\overline{\boldsymbol{v}}$ as an integral value, since it denotes an integral and not a point-wise evaluation of $\boldsymbol{v}$. The advantages of integral values are:

1 An even more extended system is, see for instance [16]

$$
\left\{\begin{array} { l l } 
{ \boldsymbol { v } - \nabla p = 0 } & { \text { in } \Omega } \\
{ \boldsymbol { u } + \mathbb { K } \boldsymbol { v } = 0 } & { \text { in } \Omega } \\
{ \nabla \cdot \boldsymbol { u } - \psi = 0 } & { \text { in } \Omega } \\
{ \psi = f } & { \text { in } \Omega }
\end{array} \quad \text { with } \quad \left\{\begin{array}{ll}
(\boldsymbol{u}, \boldsymbol{n})=\bar{u}_{n} & \text { along } \Gamma_{u} \\
p=\bar{p} & \text { along } \Gamma_{p}
\end{array}\right.\right.
$$

This seems an unnecessarily complicated system. If we eliminate $\psi$ from 5 we obtain 4 . The usefulness of this system lies in the fact that by introducing $\psi$, the conservation $\nabla \cdot \boldsymbol{u}=f$ becomes independent of the data of the PDE, in this case the right hand side function. A similar situation occurs when $\mathbb{K}=\mathbb{I}$, the identity tensor, then the equation $\boldsymbol{u}+\mathbb{K} \boldsymbol{v}=0$ in (4) seems redundant, but we have good reason to keep this seemingly redundant equation as we will show in this paper. 


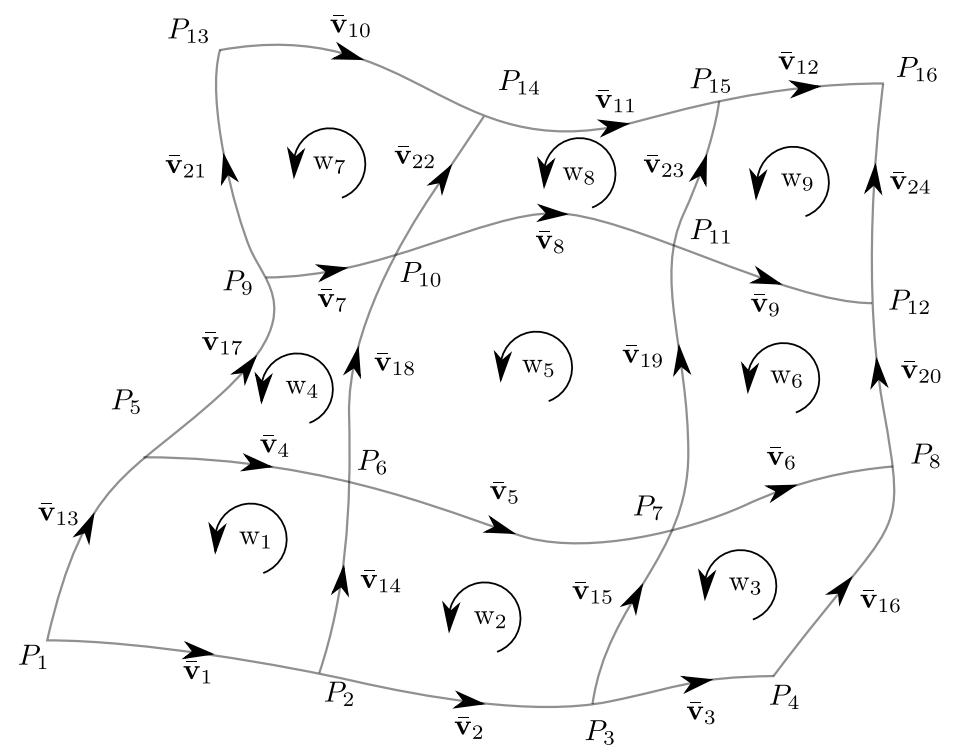

Fig. 1: Relation between pressure in points, integrated velocity along line segments and vorticity in surfaces.

1. The velocity-gradient relation is exact. It is not obtained by truncated Taylorseries expansions or does not depend on the choice of basis functions/interpolations.

2. Does not depend on mesh parameters. The mesh size $h$ does not appear in (6). Whether the curve which connects two points is straight or curved is irrelevant in this relation, therefore this relation is directly applicable on curved domains.

3. Integral quantities are additive.

Consider the points and lines segments as shown in Figure 1. In this figure the arrow along the curves indicates the direction in which $v$ is integrate ${ }^{2}$. Application of (6) shows, for instance, that

$$
\overline{\boldsymbol{v}}_{14}=P_{6}-P_{2} .
$$

The additivity property implies that

$$
\begin{aligned}
P_{7}-P_{2} & =\overline{\boldsymbol{v}}_{2}+\overline{\boldsymbol{v}}_{15}=P_{3}-P_{2}+P_{7}-P_{3} \\
& =\overline{\boldsymbol{v}}_{14}+\overline{\boldsymbol{v}}_{5}=P_{6}-P_{2}+P_{7}-P_{6},
\end{aligned}
$$

\footnotetext{
${ }^{2}$ The points in the grid shown in Figure 1 are also 'oriented', in the sense that when we 'move into a point following the integration direction' we assign a positive value and when we 'leave a point' we assign a negative value. That is why we have plus $P(B)$ and minus $P(A)$ in 6 . This is just a convention. Without loss of generality we could change this sign convention.
} 
and even more paths can be constructed that connect $P_{2}$ and $P_{7}$. The independence of the path depends critically on the assumption that the space is contractible, i.e. there are no holes in the domain (Poincaré's Lemma).

A special case is the curve from a point to itself, say $P_{2} \rightarrow P_{2}$ in Figure 1. This integral is zero and if the integral is independent of the path this implies that, for instance,

$$
0=\overline{\boldsymbol{v}}_{2}+\overline{\boldsymbol{v}}_{15}-\overline{\boldsymbol{v}}_{5}-\overline{\boldsymbol{v}}_{14}=\oint \boldsymbol{v} \cdot \mathrm{d} l=\iint \nabla \times \boldsymbol{v} \cdot \mathrm{d} \boldsymbol{S}=\mathrm{w}_{2},
$$

where we once again use the additivity property. We see that the circulation vanishes if $v$ is a potential flow, which in turn implies that the circulation of the velocity field over the boundary of any surface vanishes. Or, using Stokes' theorem, the integrated vorticity $w$ vanishes. Here the vorticity $w$ is represented as the integral over a surface.

We can collect all the integrated velocity fields and pressures in Figure 1 in the following form

$$
\left(\begin{array}{l}
\overline{\boldsymbol{v}}_{1} \\
\overline{\boldsymbol{v}}_{2} \\
\overline{\boldsymbol{v}}_{3} \\
\overline{\boldsymbol{v}}_{4} \\
\overline{\boldsymbol{v}}_{5} \\
\overline{\boldsymbol{v}}_{6} \\
\overline{\boldsymbol{v}}_{7} \\
\overline{\boldsymbol{v}}_{8} \\
\overline{\boldsymbol{v}}_{9} \\
\overline{\boldsymbol{v}}_{10} \\
\overline{\boldsymbol{v}}_{11} \\
\overline{\boldsymbol{v}}_{12} \\
\overline{\boldsymbol{v}}_{13} \\
\overline{\boldsymbol{v}}_{14} \\
\overline{\boldsymbol{v}}_{15} \\
\overline{\boldsymbol{v}}_{16} \\
\overline{\boldsymbol{v}}_{17} \\
\overline{\boldsymbol{v}}_{18} \\
\overline{\boldsymbol{v}}_{19} \\
\overline{\boldsymbol{v}}_{20} \\
\overline{\boldsymbol{v}}_{21} \\
\overline{\boldsymbol{v}}_{22} \\
\overline{\boldsymbol{v}}_{23} \\
\overline{\boldsymbol{v}}_{24}
\end{array}\right)=\left(\begin{array}{cccccccccccccccc}
-1 & 1 & 0 & 0 & 0 & 0 & 0 & 0 & 0 & 0 & 0 & 0 & 0 & 0 & 0 & 0 \\
0 & -1 & 1 & 0 & 0 & 0 & 0 & 0 & 0 & 0 & 0 & 0 & 0 & 0 & 0 & 0 \\
0 & 0 & -1 & 1 & 0 & 0 & 0 & 0 & 0 & 0 & 0 & 0 & 0 & 0 & 0 & 0 \\
0 & 0 & 0 & 0 & -1 & 1 & 0 & 0 & 0 & 0 & 0 & 0 & 0 & 0 & 0 & 0 \\
0 & 0 & 0 & 0 & 0 & -1 & 1 & 0 & 0 & 0 & 0 & 0 & 0 & 0 & 0 & 0 \\
0 & 0 & 0 & 0 & 0 & 0 & -1 & 1 & 0 & 0 & 0 & 0 & 0 & 0 & 0 & 0 \\
0 & 0 & 0 & 0 & 0 & 0 & 0 & 0 & -1 & 1 & 0 & 0 & 0 & 0 & 0 & 0 \\
0 & 0 & 0 & 0 & 0 & 0 & 0 & 0 & 0 & -1 & 1 & 0 & 0 & 0 & 0 & 0 \\
0 & 0 & 0 & 0 & 0 & 0 & 0 & 0 & 0 & 0 & -1 & 1 & 0 & 0 & 0 & 0 \\
0 & 0 & 0 & 0 & 0 & 0 & 0 & 0 & 0 & 0 & 0 & 0 & -1 & 1 & 0 & 0 \\
0 & 0 & 0 & 0 & 0 & 0 & 0 & 0 & 0 & 0 & 0 & 0 & 0 & -1 & 1 & 0 \\
0 & 0 & 0 & 0 & 0 & 0 & 0 & 0 & 0 & 0 & 0 & 0 & 0 & 0 & -1 & 1 \\
-1 & 0 & 0 & 0 & 1 & 0 & 0 & 0 & 0 & 0 & 0 & 0 & 0 & 0 & 0 & 0 \\
0 & -1 & 0 & 0 & 0 & 1 & 0 & 0 & 0 & 0 & 0 & 0 & 0 & 0 & 0 & 0 \\
0 & 0 & -1 & 0 & 0 & 0 & 1 & 0 & 0 & 0 & 0 & 0 & 0 & 0 & 0 & 0 \\
0 & 0 & 0 & -1 & 0 & 0 & 0 & 1 & 0 & 0 & 0 & 0 & 0 & 0 & 0 & 0 \\
0 & 0 & 0 & 0 & -1 & 0 & 0 & 0 & 1 & 0 & 0 & 0 & 0 & 0 & 0 & 0 \\
0 & 0 & 0 & 0 & 0 & -1 & 0 & 0 & 0 & 1 & 0 & 0 & 0 & 0 & 0 & 0 \\
0 & 0 & 0 & 0 & 0 & 0 & -1 & 0 & 0 & 0 & 1 & 0 & 0 & 0 & 0 & 0 \\
0 & 0 & 0 & 0 & 0 & 0 & 0 & -1 & 0 & 0 & 0 & 1 & 0 & 0 & 0 & 0 \\
0 & 0 & 0 & 0 & 0 & 0 & 0 & 0 & -1 & 0 & 0 & 0 & 1 & 0 & 0 & 0 \\
0 & 0 & 0 & 0 & 0 & 0 & 0 & 0 & 0 & -1 & 0 & 0 & 0 & 1 & 0 & 0 \\
0 & 0 & 0 & 0 & 0 & 0 & 0 & 0 & 0 & 0 & -1 & 0 & 0 & 0 & 1 & 0 \\
0 & 0 & 0 & 0 & 0 & 0 & 0 & 0 & 0 & 0 & 0 & -1 & 0 & 0 & 0 & 1
\end{array}\right)\left(\begin{array}{c} 
\\
P_{1} \\
P_{2} \\
P_{3} \\
P_{4} \\
P_{5} \\
P_{6} \\
P_{7} \\
P_{8} \\
P_{9} \\
P_{10} \\
P_{11} \\
P_{12} \\
P_{13} \\
P_{14} \\
P_{15} \\
P_{16}
\end{array}\right) .
$$

If we store all $\overline{\boldsymbol{v}}_{i}$ in a vector $\mathrm{v}$ and all $P_{j}$ in a vector $\mathrm{P}$ and denote the matrix by $\mathbb{E}^{1,0}$, we have

$$
\mathrm{v}=\mathbb{E}^{1,0} \mathrm{P} .
$$

If we now also collect all the integrated vorticities, $w_{i}$, we can relate them to the integrated velocities in the following way 


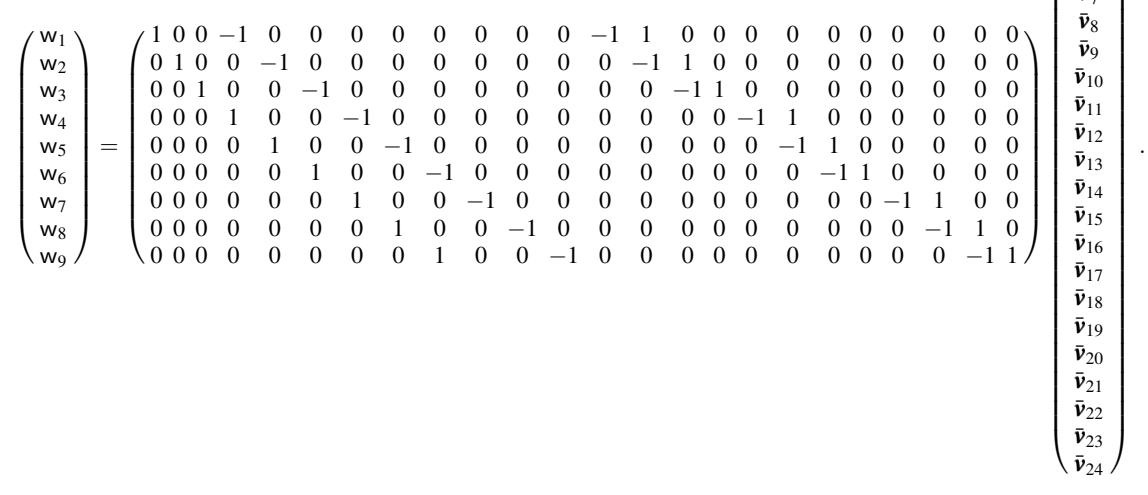

If we store all vorticity integrals, $w_{i}$ in the vector $w$, then we can write this as

$$
\mathrm{w}=\mathbb{E}^{2,1} \mathrm{v} .
$$

The matrices $\mathbb{E}^{1,0}$ and $\mathbb{E}^{2,1}$ are called incidence matrices. We have $\mathbb{E}^{2,1} \cdot \mathbb{E}^{1,0} \equiv 0$. This identity holds for this particular case, but is generally true; it holds when we would have used triangles or polyhedra instead of quadrilaterals and it holds in any space dimension $d$. If $\mathbb{E}^{1,0}$ represents the gradient operation and $\mathbb{E}^{2,1}$ represents the curl operation, then $\mathbb{E}^{2,1} \cdot \mathbb{E}^{1,0} \equiv 0$ is the discrete analogue of the vector identity $\nabla \times \nabla \equiv 0,[24,-27,49,88,110]$.

If boundary conditions for $p$ are prescribed along $\partial \Omega$, then these degrees of freedom can be removed from the grid in Figure 1

If $p$ is known along the boundary then the integral of $v$ is also known along the boundary, so the degrees of freedom for $v$ can also be removed. Relabeling the remaining unknowns gives the geometric degrees of freedom as shown in Figure 2

$$
\left(\begin{array}{l}
\overline{\boldsymbol{v}}_{1} \\
\overline{\boldsymbol{v}}_{2} \\
\overline{\boldsymbol{v}}_{3} \\
\overline{\boldsymbol{v}}_{4} \\
\overline{\boldsymbol{v}}_{5} \\
\overline{\boldsymbol{v}}_{6} \\
\overline{\boldsymbol{v}}_{7} \\
\overline{\boldsymbol{v}}_{8} \\
\overline{\boldsymbol{v}}_{9} \\
\overline{\boldsymbol{v}}_{10} \\
\overline{\boldsymbol{v}}_{11} \\
\overline{\boldsymbol{v}}_{12}
\end{array}\right)=\left(\begin{array}{cccc}
1 & 0 & 0 & 0 \\
-1 & 1 & 0 & 0 \\
0 & -1 & 0 & 0 \\
0 & 0 & 1 & 0 \\
0 & 0 & -1 & 1 \\
0 & 0 & 0 & -1 \\
1 & 0 & 0 & 0 \\
0 & 1 & 0 & 0 \\
-1 & 0 & 1 & 0 \\
0 & -1 & 0 & 1 \\
0 & 0 & -1 & 0 \\
0 & 0 & 0 & -1
\end{array}\right)\left(\begin{array}{l}
P_{1} \\
P_{2} \\
P_{3} \\
P_{4}
\end{array}\right)
$$




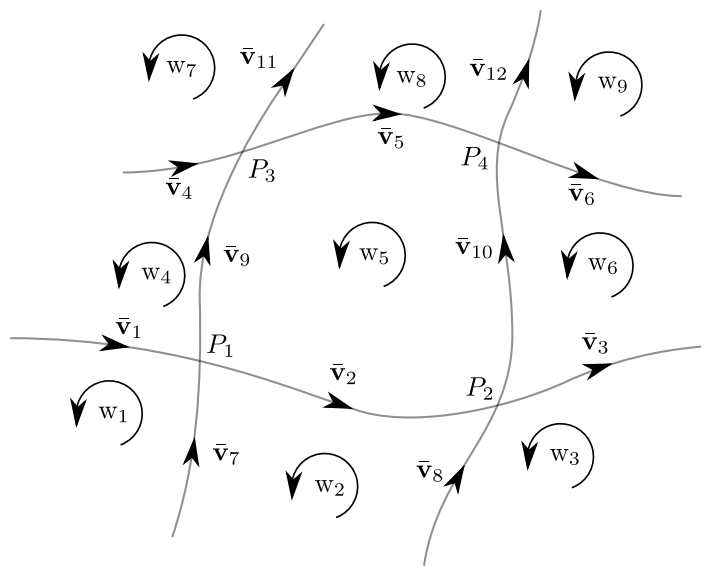

Fig. 2: Relation between pressure in points and integrated velocity along line segments in case $\Gamma_{p}=\partial \Omega$.

\subsection{Divergence relation}

Consider a bounded, contractible volume $\mathscr{V} \subset \Omega$ then we have

$$
\nabla \cdot \boldsymbol{u}=f \quad \Longrightarrow \quad \int_{\partial \mathscr{V}} \boldsymbol{u} \cdot \boldsymbol{n} \mathrm{d} S=\int_{\mathscr{V}} f \mathrm{~d} \mathscr{V}
$$

If the boundary $\partial \mathscr{V}$ can be partitioned into $n$ sub-boundaries, $\partial \mathscr{V}=\bigcup_{i} \Gamma_{i}$ and $\bigcap_{i} \Gamma_{i}=0$, we have

$$
\pm \sum_{i=1}^{n} \overline{\boldsymbol{u}}_{i}= \pm \sum_{i=1}^{n} \int_{\Gamma_{i}} \boldsymbol{u} \cdot \boldsymbol{n} \mathrm{d} S=\int_{\mathscr{V}} f \mathrm{~d} \mathscr{V}=: f_{\mathscr{V}}
$$

where we have the convention that the fluxes, $\overline{\boldsymbol{u}}_{i}$, are positive when the flow leaves the volume and negative when the flow enters the volume. For a 2D case the integral flux degrees of freedom, $\overline{\boldsymbol{u}}_{i}$ are depicted in Figure 3 . The arrow in this figure indicates the positive default direction of the fluxes. The integrated values of source function $f$ are shown in the 2D volumes in Figure 3 as $f_{i}$. The topological relation between the fluxes and the integrated source values $f_{i}$, for the situation shown in Figure 3 , is given by 


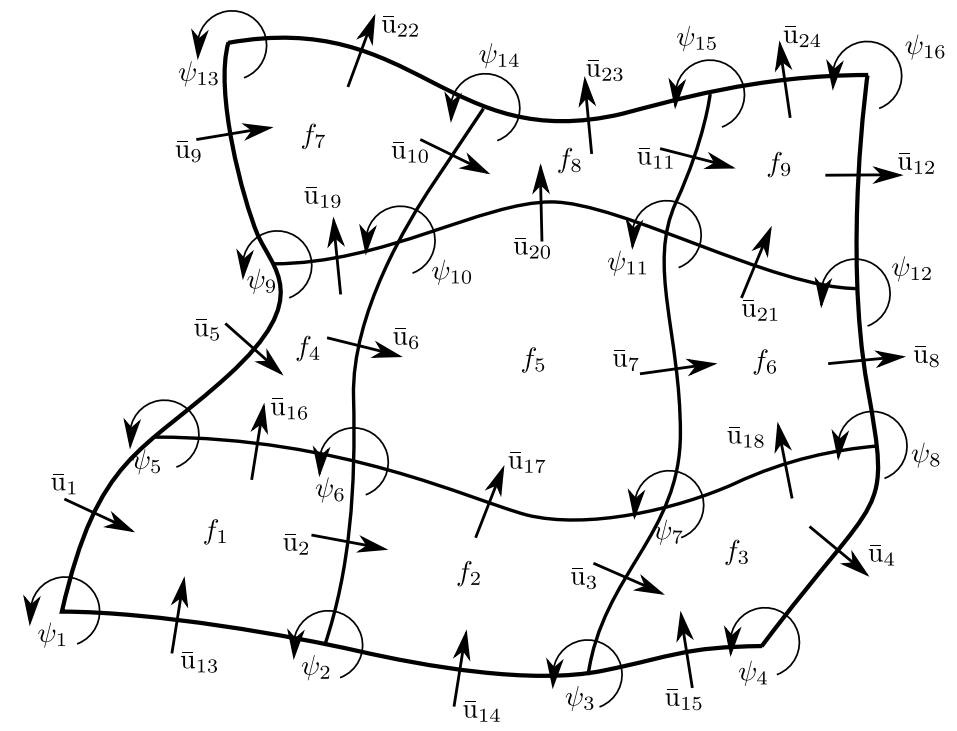

Fig. 3: Stream function, fluxes and the divergence degrees of freedom.

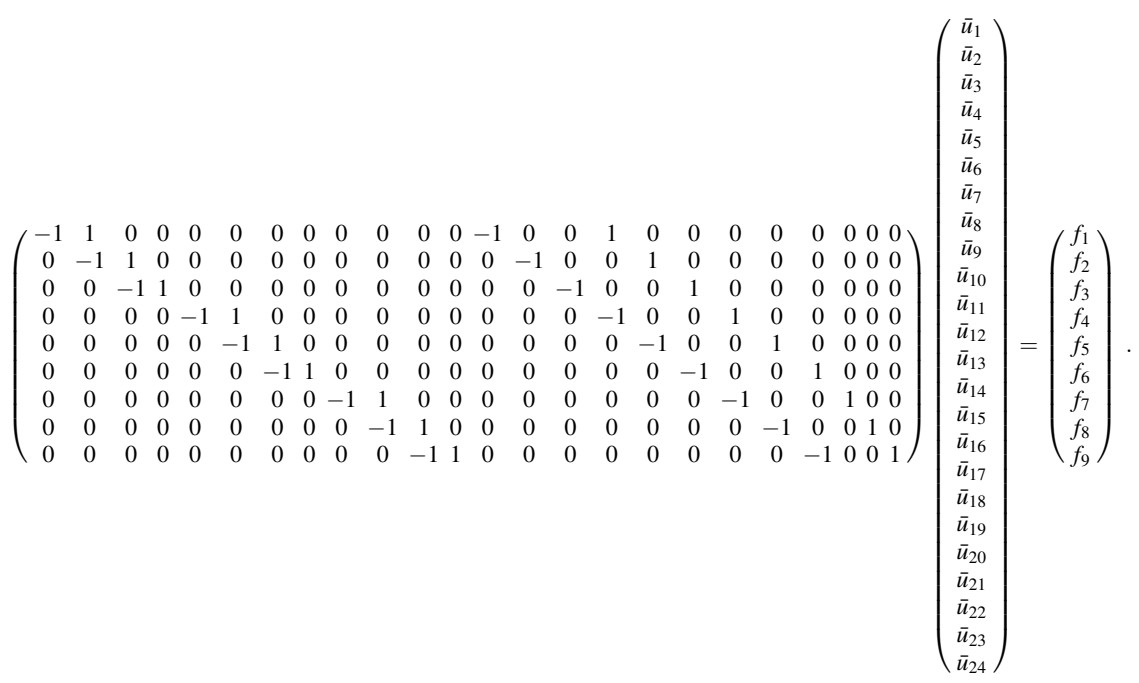

Collecting all fluxes and source terms in vectors $u$ and $f$, respectively, we can write this equation as

$$
\tilde{\mathbb{E}}^{2,1} \mathrm{u}=\mathrm{f} .
$$

The matrix $\widetilde{\mathbb{E}}^{2,1}$ is the incidence matrix which represents the divergence operator, not to be confused with $\mathbb{E}^{2,1}$ in $(8)$ which represents the curl operator.

If, in the $2 \mathrm{D}$ case, the flow field is divergence-free, i.e. $f=0$, we know that a stream function $\boldsymbol{\psi}$ exists which is connected to $\boldsymbol{u}$ by 


$$
u_{x}=\frac{\partial \boldsymbol{\psi}}{\partial y}, \quad u_{y}=-\frac{\partial \boldsymbol{\psi}}{\partial x} .
$$

If we represent the stream function in the nodes of the grid shown in Figure 3 then we have the exact topological equation

$$
\left(\begin{array}{l}
\bar{u}_{1} \\
\bar{u}_{2} \\
\bar{u}_{3} \\
\bar{u}_{4} \\
\bar{u}_{5} \\
\bar{u}_{6} \\
\bar{u}_{7} \\
\bar{u}_{8} \\
\bar{u}_{9} \\
\bar{u}_{10} \\
\bar{u}_{11} \\
\bar{u}_{12} \\
\bar{u}_{13} \\
\bar{u}_{14} \\
\bar{u}_{15} \\
\bar{u}_{16} \\
\bar{u}_{17} \\
\bar{u}_{18} \\
\bar{u}_{19} \\
\bar{u}_{20} \\
\bar{u}_{21} \\
\bar{u}_{22} \\
\bar{u}_{23} \\
\bar{u}_{24}
\end{array}\right) \quad\left(\begin{array}{cccccccccccccccc}
-1 & 0 & 0 & 0 & 1 & 0 & 0 & 0 & 0 & 0 & 0 & 0 & 0 & 0 & 0 & 0 \\
0 & -1 & 0 & 0 & 0 & 1 & 0 & 0 & 0 & 0 & 0 & 0 & 0 & 0 & 0 & 0 \\
0 & 0 & -1 & 0 & 0 & 0 & 1 & 0 & 0 & 0 & 0 & 0 & 0 & 0 & 0 & 0 \\
0 & 0 & 0 & -1 & 0 & 0 & 0 & 1 & 0 & 0 & 0 & 0 & 0 & 0 & 0 & 0 \\
0 & 0 & 0 & 0 & -1 & 0 & 0 & 0 & 1 & 0 & 0 & 0 & 0 & 0 & 0 & 0 \\
0 & 0 & 0 & 0 & 0 & -1 & 0 & 0 & 0 & 1 & 0 & 0 & 0 & 0 & 0 & 0 \\
0 & 0 & 0 & 0 & 0 & 0 & -1 & 0 & 0 & 0 & 1 & 0 & 0 & 0 & 0 & 0 \\
0 & 0 & 0 & 0 & 0 & 0 & 0 & -1 & 0 & 0 & 0 & 1 & 0 & 0 & 0 & 0 \\
0 & 0 & 0 & 0 & 0 & 0 & 0 & 0 & -1 & 0 & 0 & 0 & 1 & 0 & 0 & 0 \\
0 & 0 & 0 & 0 & 0 & 0 & 0 & 0 & 0 & -1 & 0 & 0 & 0 & 1 & 0 & 0 \\
0 & 0 & 0 & 0 & 0 & 0 & 0 & 0 & 0 & 0 & -1 & 0 & 0 & 0 & 1 & 0 \\
0 & 0 & 0 & 0 & 0 & 0 & 0 & 0 & 0 & 0 & 0 & -1 & 0 & 0 & 0 & 1 \\
1 & -1 & 0 & 0 & 0 & 0 & 0 & 0 & 0 & 0 & 0 & 0 & 0 & 0 & 0 & 0 \\
0 & 1 & -1 & 0 & 0 & 0 & 0 & 0 & 0 & 0 & 0 & 0 & 0 & 0 & 0 & 0 \\
0 & 0 & 1 & -1 & 0 & 0 & 0 & 0 & 0 & 0 & 0 & 0 & 0 & 0 & 0 & 0 \\
0 & 0 & 0 & 0 & 1 & -1 & 0 & 0 & 0 & 0 & 0 & 0 & 0 & 0 & 0 & 0 \\
0 & 0 & 0 & 0 & 0 & 1 & -1 & 0 & 0 & 0 & 0 & 0 & 0 & 0 & 0 & 0 \\
0 & 0 & 0 & 0 & 0 & 0 & 1 & -1 & 0 & 0 & 0 & 0 & 0 & 0 & 0 & 0 \\
0 & 0 & 0 & 0 & 0 & 0 & 0 & 0 & 1 & -1 & 0 & 0 & 0 & 0 & 0 & 0 \\
0 & 0 & 0 & 0 & 0 & 0 & 0 & 0 & 0 & 1 & -1 & 0 & 0 & 0 & 0 & 0 \\
0 & 0 & 0 & 0 & 0 & 0 & 0 & 0 & 0 & 0 & 1 & -1 & 0 & 0 & 0 & 0 \\
0 & 0 & 0 & 0 & 0 & 0 & 0 & 0 & 0 & 0 & 0 & 0 & 1 & -1 & 0 & 0 \\
0 & 0 & 0 & 0 & 0 & 0 & 0 & 0 & 0 & 0 & 0 & 0 & 0 & 1 & -1 & 0 \\
0 & 0 & 0 & 0 & 0 & 0 & 0 & 0 & 0 & 0 & 0 & 0 & 0 & 0 & 1 & -1
\end{array}\right)\left(\begin{array}{l} 
\\
\\
\psi_{1} \\
\psi_{2} \\
\psi_{3} \\
\psi_{4} \\
\psi_{5} \\
\psi_{6} \\
\psi_{7} \\
\psi_{8} \\
\psi_{9} \\
\psi_{10} \\
\psi_{11} \\
\psi_{12} \\
\psi_{13} \\
\psi_{14} \\
\psi_{15} \\
\psi_{16}
\end{array}\right) .
$$

We can write this in terms of incidence matrices a 3

$$
\mathrm{u}=\tilde{\mathbb{E}}^{1,0} \psi .
$$

If the flux $\boldsymbol{u}$ is prescribed along the $\Gamma_{u}$ the associated edges (2D) or surfaces (3D) can be eliminated from the system $\mathbb{E}^{d, d-1} \mathrm{u}=\mathrm{f}$ and transferred to the right hand side.

For the discretization of (4) the first and last equation in that system can be represented on the mesh by

${ }^{3}$ Note that if we performed the same steps in $3 \mathrm{D}$, then the divergence relation 10 would be

$$
\tilde{\mathbb{E}}^{3,2} \mathbf{u}=\mathbf{f},
$$

and the 2D stream function becomes the 3D stream vector field and we would have

$$
\mathrm{u}=\tilde{\mathbb{E}}^{2,1} \psi
$$

So clearly the incidence matrices $\tilde{\mathbb{E}}$ depend on the dimension of the space $d$ in which the problem is posed. Note that this is not the case for the incidence matrices $\mathbb{E}$. Alternatively, we could refer to the dimension-dependent incidence matrices as

$$
\mathbb{E}^{d, d-1}=\left\{\begin{array}{ll}
\tilde{\mathbb{E}}^{2,1} & \text { if } d=2 \\
\tilde{\mathbb{E}}^{3,2} & \text { if } d=3
\end{array} \text { and } \quad \mathbb{E}^{d-1, d-2}=\left\{\begin{array}{ll}
\tilde{\mathbb{E}}^{1,0} & \text { if } d=2 \\
\tilde{\mathbb{E}}^{2,1} & \text { if } d=3
\end{array},\right.\right.
$$

in which case it is immediately clear that these matrices depend on the $d$. From now on we will use the incidence matrices with the $d$, because then the results are valid for any space dimension $d$. 


$$
\left\{\begin{array}{l}
\mathrm{v}-\mathbb{E}^{1,0} \mathrm{p}=0 \\
\tilde{\mathbb{E}}^{d, d-1} \mathrm{u}=\mathrm{f}
\end{array} .\right.
$$

Prescription of boundary conditions $p$ along $\Gamma_{u}$ and $u$ along $\Gamma_{u}$ can be done strongly. The degrees of freedom can be eliminated and transferred to the right hand side. The equation between $p$ and $v$ is exact on any grid and the discrete divergence relation between $\boldsymbol{u}$ and $f$ is exact on any grid. Note the $(\boldsymbol{v}, p)$-grid is not necessarily the $(\boldsymbol{u}, f)$-grid, so in principle we can use different grids for both equations.

Unfortunately, neither of the two problems, $\boldsymbol{v}=\nabla p$ and $\nabla \cdot \boldsymbol{u}=f$ has a unique solution on their respective grids. It is the final equation in (4), $\boldsymbol{u}=-\mathbb{K} \boldsymbol{v}$, that couples the solution on the two grids and renders a unique solution. It is also in this equation that the numerical approximation is made; the more accurate we approximate this algebraic equation, the more accurate the solution to the first order system (4) will be.

For many numerical method $\$^{4}$ well-posedness requires that the number of discrete degrees of freedom $\overline{\boldsymbol{v}}_{i}$ is equal to the discrete number of degrees of freedom $\overline{\boldsymbol{u}}_{j}$, or more geometrically, that the number of $k$-dimensional geometric objects on one grid is equal to the number of $(d-k)$-dimensional geometric objects on the other grid. Here $k=0$ refers to points in the grid, $k=1$ to edges in the grid, $k=2$ the faces in the grid, and $k=3$ the volumes in the grid.

The requirement $\# k=\#(d-k)$ cannot be accomplished on a single grid, so this requires two different grids which are constructed in such a way that $\# k=\#(d-k)$ holds, [24, 25, 49, 82, 88, 110].

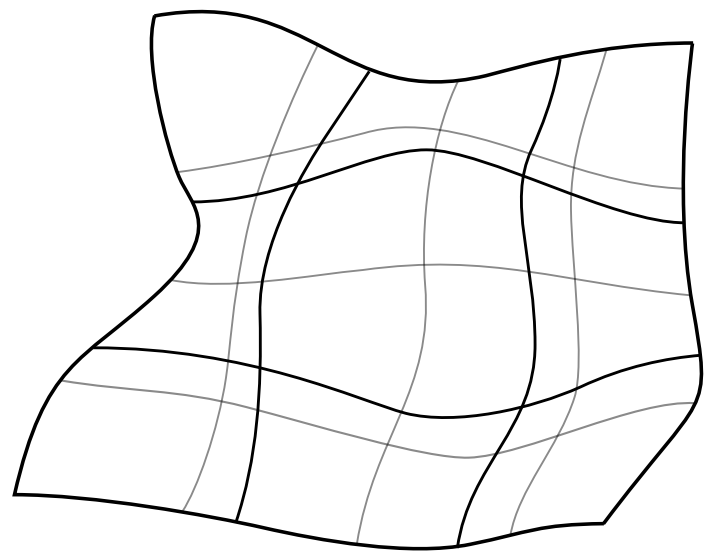

Fig. 4: The primal grid (thin gray) where $(\boldsymbol{v}, p)$ are represented and the dual grid (thick black) where $(\boldsymbol{u}, f)$ are represented. Note that $\Gamma_{p}=\partial \Omega$ and consequently $\Gamma_{u}=\varnothing$.

\footnotetext{
${ }^{4}$ A notable exception is the class of least-squares formulations which aims to minimize the expression $\boldsymbol{u}+\mathbb{K} \boldsymbol{v}$ [17].
} 
A dual grid complex is shown in Figure 4. The integral quantities $(\boldsymbol{v}, p)$ can be represented on the gray grid. If $p$ is prescribed along the entire boundary, then those degrees of freedom are eliminated (including the gray edges along the boundary for which the integral value $v$ is then known also), see for instance Figure 2 In that case flux $\boldsymbol{u}$ along the boundary cannot be prescribed. In Figure 4, the number of points in the gray grid, 9, equals the number of surfaces in the black grid, the number of edges in the grey grid is equal to the number of edges on the black grid, 24, and the number of surfaces on the gray grid equals the number of points in the black grid, 16 , therefore, we have $\# k=\#(d-k)$ for $d=2$.

Alternatively, we could have represented $(\boldsymbol{u}, f)$ on the gray grid with $\boldsymbol{u}$ and the stream function $\psi$ prescribed and $(\boldsymbol{v}, p)$ on the black grid.In this case $\Gamma_{u}=\partial \Omega$ and $\Gamma_{p}=\varnothing$.

\subsection{Dual grids}

If dual grids, such as described above, are employed then we have two properties:

1. There exists a square, invertible matrix $\mathbb{H}_{\mathbb{K}}^{d-1,1}$ such that $\mathrm{u}=\mathbb{H}_{\mathbb{K}}^{d-1,1} \mathrm{v}$.

2. The incidence matrices on the primal and dual grid satisfy ${ }^{5}$

$$
\mathbb{E}^{d-k, d-k-1}=\left(\mathbb{E}^{k, k-1}\right)^{T}
$$

If we use dual grids and these properties hold, we can write (4) as

$$
\left\{\begin{array}{l}
\mathrm{v}-\mathbb{E}^{1,0} \mathrm{p}=0 \\
\mathrm{u}-\mathbb{H}_{\mathbb{K}}^{d-1,1} \mathrm{v}=0 \\
\mathbb{E}^{1,0^{T}} \mathrm{u}=\mathrm{f}
\end{array}\right.
$$

where the vectors $p, v, u$ and $f$ contain the integral quantities in the mesh as discussed in the previous sections.

In the diagram below, we place the various integral values in appropriate 'spaces'

$$
\begin{aligned}
& \mathrm{p} \in H_{0} \stackrel{\mathbb{E}^{1,0}}{\longrightarrow} \mathrm{v} \in H_{1} \stackrel{\mathbb{E}^{2,1}}{\longrightarrow} \xi \in H_{2} \\
& \mathbb{\mathbb { H } ^ { d , 0 }} \| \mathbb{H}_{\mathbb{H}^{0, d}} \quad \mathbb{H}_{\mathbb{K}}^{d-1,1} \mid \mathbb{H}_{\mathbb{K}^{-1}}^{1, d-1} \\
& \mathrm{f} \in \tilde{H}_{d} \underset{\mathbb{E}^{1,0^{T}}}{\longleftarrow} \mathrm{u} \in \tilde{H}_{d-1} \underset{\mathbb{E}^{2,1^{T}}}{\longleftarrow} \psi \in \tilde{H}_{d-2}
\end{aligned}
$$

\footnotetext{
5 This relation is true if the orientations on primal and dual grid agree. This is not always the case and then the relation reads $\mathbb{E}^{d-k, d-k-1}=-\mathbb{E}^{k, k-1^{T}}$. A well known example is the duality between grad and div.
} 
Here $H_{k}$ denotes the space of values assigned to $k$-dimensional objects in the $H$-grid for $k=0,1,2$. If $\tilde{H}$ denotes the dual grid, then $\tilde{H}_{l}$ is the space of values assigned to $l$-dimensional objects in the $\tilde{H}$-grid.

For dual grids the number of points in the $H$-grid is equal to the number of $d$ dimensional volumes in the dual grid $\tilde{H}$. Let $\mathbb{H}^{d, 0}$ and $\mathbb{H}^{0, d}$ be square, invertible matrices which map between $H_{0}$ and $\tilde{H}_{d}$ as shown in the diagram above.

If we eliminate $v$ and $u$ from (12) we have

$$
\mathbb{E}^{1,0^{T}} \mathbb{H}_{\mathbb{K}}^{d-1,1} \mathbb{E}^{1,0} \mathrm{p}=\mathrm{f} .
$$

This discretization corresponds to (2). We will refer to this formulation as the direct formulation.

If $\mathrm{p} \in \tilde{H}_{d}$ we can set up the diffusion problem as

$$
\left\{\begin{array}{ll}
-\mathbb{H}_{\mathbb{K}}^{1, d-1} \mathrm{u}+\mathbb{E}^{d, d-1^{T}} \mathbb{H}^{0, d} \mathrm{p} & =0 \\
\mathbb{H}^{0, d} \mathbb{E}^{d, d-1} \mathrm{u} & =\mathrm{f}
\end{array} .\right.
$$

This formulation, where we solve for $p$ and $\boldsymbol{u}$ simultaneously, resembles (3), and will be called the mixed formulation, [35].

\section{Mimetic spectral element method}

The incidence matrices introduced in the previous section are generic and only depend on the grid topology. The matrices $\mathbb{H}$ which switch between the primal and the dual grid representation explicitly depend on the numerical method that is used. In this section we will introduce spectral element functions which interpolate the integral values in a grid. With these functions we can construct the $\mathbb{H}$-matrices, which turn out to be (weighted) finite element mass matrices. The derivation in this section will be on an orthogonal grid. The extension to curvilinear grids will be discussed in the next section.

\subsection{One dimensional spectral basis functions}

Consider the interval $[-1,1] \subset \mathbb{R}$ and the Legendre polynomials, $L_{N}(\xi)$, of degree $N, \xi \in[-1,1]$. The $(N+1)$ roots, $\xi_{i}$, of the polynomial $\left(1-\xi^{2}\right) L_{N}^{\prime}(\xi)$ satisfy $-1 \leq$ $\xi_{i} \leq 1$. Here $L_{N}^{\prime}(\xi)$ is the derivative of the Legendre polynomial. The roots $\xi_{i}, i=$ $0, \ldots, N$, are called the Gauss-Lobatto-Legendre (GLL) points, [41]. Let $h_{i}(\xi)$ be the Lagrange polynomial through the GLL points such that 


$$
h_{i}\left(\xi_{j}\right)=\left\{\begin{array}{ll}
1 & \text { if } i=j \\
0 & \text { if } i \neq j
\end{array} \quad i, j=0, \ldots N .\right.
$$

The explicit form of the Lagrange polynomials in terms of the Legendre polynomials is given by

$$
h_{i}(\xi)=\frac{\left(1-\xi^{2}\right) L_{N}^{\prime}(\xi)}{N(N+1) L_{N}\left(\xi_{i}\right)\left(\xi_{i}-\xi\right)} .
$$

Let $f(\xi)$ be a function defined for $\xi \in[-1,1]$ by

$$
f(\xi)=\sum_{i=0}^{N} a_{i} h_{i}(\xi)
$$

Using property 15 we see that $f\left(\xi_{j}\right)=a_{j}$, so the expansion coefficients in 17] coincide with the value of $f$ in the GLL nodes. We will refer to this expansion as a nodal expansion, because the expansion coefficients, $a_{i}$ in $(17)$ are the value of $f(\xi)$ in the nodes $\xi_{i}$. The basis functions $h_{i}(\xi)$ are polynomials of degree $N$.

From the nodal basis functions, define the polynomials $e_{i}(\xi)$ by

$$
e_{i}(\xi)=-\sum_{k=0}^{i-1} \frac{d h_{k}(\xi)}{d \xi}
$$

The functions $e_{i}(\xi)$ are polynomials of degree $(N-1)$. These polynomials satisfy, [61, 82, 96]

$$
\int_{\xi_{j-1}}^{\xi_{j}} e_{i}(\xi)=\left\{\begin{array}{ll}
1 & \text { if } i=j \\
0 & \text { if } i \neq j
\end{array} \quad i, j=1, \ldots N .\right.
$$

Let a function $g(\xi)$ be expanded in these functions

$$
g(\xi)=\sum_{i=1}^{N} b_{i} e_{i}(\xi)
$$

then using 19

$$
\int_{\xi_{j-1}}^{\xi_{j}} g(\xi)=b_{j} .
$$

So the expansion coefficients $b_{i}$ in 20 coincide with the integral of $g$ over the edge $\left[\xi_{i-1}, \xi_{i}\right]$. We will call these basis functions edge functions and refer to the expansion [20] as an edge expansion, see for instance [16, 82, 96] for examples of nodal and edge expansions. 
Let $f(\xi)$ be expanded in terms Lagrange polynomials as in (17), then the derivative ${ }^{6}$ of $f$ is given by, [61, 82, 96]

$$
f^{\prime}(\xi)=\sum_{i=0}^{N} a_{i} h_{i}^{\prime}(\xi)=\sum_{i=1}^{N}\left(a_{i}-a_{i-1}\right) e_{i}(\xi)
$$

If we collect all the expansion coefficients in a column vector and all the basis functions in a row vector we have

$$
f(\xi)=\left[\begin{array}{llll}
h_{0} & h_{1} & \ldots & h_{N}
\end{array}\right]\left[\begin{array}{c}
a_{0} \\
\vdots \\
a_{N}
\end{array}\right],
$$

then the derivative is given by 21

$$
f^{\prime}(\xi)=\left[\begin{array}{lll}
e_{1} & \ldots & e_{N}
\end{array}\right]\left(\begin{array}{cccccc}
-1 & 1 & 0 & \ldots & & 0 \\
& \ddots & \ddots & & & 0 \\
& & -1 & 1 & & 0 \\
& & & \ddots & \ddots & \\
0 & & \ldots & 0 & -1 & 1
\end{array}\right)\left[\begin{array}{c}
a_{0} \\
\vdots \\
a_{N}
\end{array}\right]=\left[\begin{array}{lll}
e_{1} & \ldots & e_{N}
\end{array}\right] \mathbb{E}^{1,0}\left[\begin{array}{c}
a_{0} \\
\\
\vdots \\
a_{N}
\end{array}\right]
$$

So taking the derivative essentially consists of two step: Apply the matrix $\mathbb{E}^{1,0}$ to the expansion coefficients and expand in a new basis.

\subsection{Two dimensional expansions}

\subsubsection{Expanding $p$ (Direct formulation)}

In finite element methods the direct finite element formulation for the anisotropic diffusion problem is given by: For $(\mathbb{K} \nabla p, \boldsymbol{n})=0$ along $\Gamma_{u}$ and $f \in H^{-1}(\Omega)$, find $p \in H_{0, \Gamma_{p}}^{1}(\Omega)$ such that

\footnotetext{
${ }^{6}$ Note that the set of polynomials $\left\{h_{i}^{\prime}\right\}, i=0, \ldots, N$ is linearly dependent and therefore does not form a basis, while the set $\left\{e_{i}\right\}, i=1, \ldots, N$ is linearly independent and therefore forms a basis for the derivatives of the nodal expansion (17).

${ }^{7}$ The matrix $\mathbb{E}^{1,0}$ is the incidence matrix as was discussed in Sections 2.1 and 2.2 It takes the nodal expansion coefficients and maps them to the edge expansion coefficients. The incidence matrix is the topological part of the derivative. It is independent of the order of the method (the polynomial degree $\mathrm{N}$ ) and the size or the shape of the mesh. The incidence matrix only depends on the topology and orientation of the grid, see [18, 81, 82 .
} 


$$
(\nabla \tilde{p}, \mathbb{K} \nabla p)=(\tilde{p}, f), \quad \forall \tilde{p} \in H_{0, \Gamma_{p}}^{1}(\Omega) .
$$

where $H_{0, \Gamma_{p}}^{1}=\left\{p \in H^{1}(\Omega) \mid p=0\right.$ on $\left.\Gamma_{p}\right\}$.

Consider $[-1,1]^{2} \subset \mathbb{R}^{2}$ and let $p(\xi, \eta)$ be expanded as

$$
p(\xi, \eta)=\sum_{i=0}^{N} \sum_{j=0}^{N} p_{i, j} h_{i}(\xi) h_{j}(\eta) .
$$

From $\left[15\right.$ it follows that $p_{i, j}=p\left(\xi_{i}, \eta_{j}\right)$. If we take the gradient of $p$ using 21] we have

$$
\begin{aligned}
& \nabla p=\left(\begin{array}{l}
\sum_{i=1}^{N} \sum_{j=0}^{N}\left(p_{i, j}-p_{i-1, j}\right) e_{i}(\xi) h_{j}(\eta) \\
\sum_{i=0}^{N} \sum_{j=1}^{N}\left(p_{i, j}-p_{i, j-1}\right) h_{i}(\xi) e_{j}(\eta)
\end{array}\right)
\end{aligned}
$$

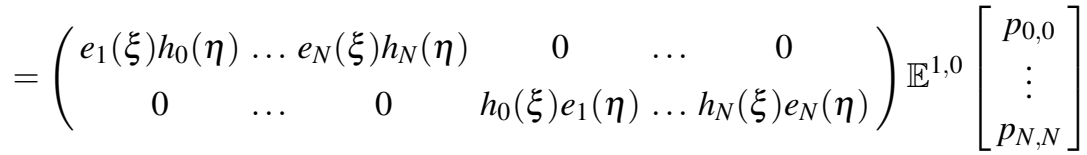

$$
\begin{aligned}
& =\left(\begin{array}{ccccc}
e_{1}(\xi) h_{0}(\eta) & \ldots e_{N}(\xi) h_{N}(\eta) & 0 & \ldots & 0 \\
0 & \ldots & 0 & h_{0}(\xi) e_{1}(\eta) & \ldots h_{N}(\xi) e_{N}(\eta)
\end{array}\right) \mathbb{E}^{1,0} \mathrm{p} .
\end{aligned}
$$

If we insert this in 24], we have

$$
\left(\mathbb{E}^{1,0}\right)^{T} \mathbb{M}_{\mathbb{K}}^{(1)} \mathbb{E}^{1,0} \mathrm{p}=\mathrm{f},
$$

where

$$
\mathbb{M}_{\mathbb{K}}^{(1)}=\iint_{\Omega}\left(\begin{array}{cc}
e_{1}(\xi) h_{0}(\eta) & 0 \\
\vdots & \vdots \\
e_{N}(\xi) h_{N}(\eta) & 0 \\
0 & h_{0}(\xi) e_{1}(\eta) \\
\vdots & \vdots \\
0 & h_{N}(\xi) e_{N}(\eta)
\end{array}\right) \mathbb{K}\left(\begin{array}{ccccc}
e_{1}(\xi) h_{0}(\eta) \ldots e_{N}(\xi) h_{N}(\eta) & 0 & \ldots & 0 \\
0 & \ldots & 0 & h_{0}(\xi) e_{1}(\eta) \ldots h_{N}(\xi) e_{N}(\eta)
\end{array}\right) \mathrm{d} \Omega
$$

and $\mathrm{p}$ is the vector which contains the expansion coefficients of $p(\xi, \eta)$ in 25 .

The vector $f$ in 28 is given by

$$
\mathrm{f}=\iint_{\Omega}\left(\begin{array}{c}
h_{0}(\xi) h_{0}(\eta) \\
\vdots \\
h_{N}(\xi) h_{N}(\eta)
\end{array}\right) f(\xi, \eta) \mathrm{d} \Omega .
$$

If we compare 28, with $\sqrt{13}$, we see that the $\mathbb{H}_{\mathbb{K}}^{d-1,1}$-matrix from 13 is represented in the finite element formulation by the weighted mass matrix $\mathbb{M}_{\mathbb{K}}^{(1)}$ given by [29], see also [18, 115]. 


\subsubsection{Expanding $u$ and $p$ (Mixed formulation)}

The mixed formulation for the anisotropic steady diffusion problem is given by: For $p=0$ along $\Gamma_{p}$ and for $f \in L^{2}(\Omega)$, find $u \in H_{0, \Gamma_{n}}(\operatorname{div} ; \Omega)$ such that

$$
\left\{\begin{array}{rlrl}
-\left(\tilde{\boldsymbol{u}}, \mathbb{K}^{-1} \boldsymbol{u}\right)+(\nabla \cdot \tilde{\boldsymbol{u}}, p) & =0 & & \forall \tilde{\boldsymbol{u}} \in H_{0, \Gamma_{u}}(\operatorname{div} ; \Omega) \\
(\tilde{p}, \nabla \cdot \boldsymbol{u}) & =f & & \forall \tilde{p} \in L^{2}(\Omega)
\end{array} .\right.
$$

where, $H_{0, \Gamma_{u}}(\operatorname{div} ; \Omega)=\left\{u \in H(\operatorname{div} ; \Omega) \mid u \cdot n=0\right.$ along $\left.\Gamma_{u}\right\}$.

In contrast to the pressure expansion in Section 3.2.1 in the direct formulation, 25], in the mixed formulation the pressure is expanded in terms of edge functions

$$
p(\xi, \eta)=\sum_{i=1}^{N} \sum_{j=1}^{N} p_{i, j} e_{i}(\xi) e_{j}(\eta)
$$

The velocity $\boldsymbol{u}$ is expanded as

$$
\begin{aligned}
& \boldsymbol{u}=\left(\begin{array}{c}
u \\
v
\end{array}\right)=\left(\begin{array}{l}
\sum_{i=0}^{N} \sum_{j=1}^{N} u_{i, j} h_{i}(\xi) e_{j}(\eta) \\
\sum_{i=1}^{N} \sum_{j=0}^{N} v_{i, j} e_{i}(\xi) h_{j}(\eta)
\end{array}\right) \\
& =\left(\begin{array}{cccccc}
h_{0}(\xi) e_{1}(\eta) & \ldots & h_{N}(\xi) e_{N}(\eta) & 0 & \ldots & 0 \\
0 & \ldots & 0 & e_{1}(\xi) h_{0}(\eta) & \ldots & e_{N}(\xi) h_{N}(\eta)
\end{array}\right)\left(\begin{array}{c}
u_{0,1} \\
\vdots \\
u_{N, N} \\
v_{1,0} \\
\vdots \\
v_{N, N}
\end{array}\right) .
\end{aligned}
$$

Application of the divergence operator to (32) and using 21] we obtain

$$
\begin{aligned}
\nabla \cdot \boldsymbol{u} & =\sum_{i=1}^{N} \sum_{j=1}^{N}\left(u_{i, j}-u_{i-1, j}+v_{i, j}-v_{i, j-1}\right) e_{i}(\xi) e_{j}(\eta) \\
& =\left(e_{1}(\xi) e_{1}(\eta) \ldots e_{N}(\xi) e_{N}(\eta)\right) \mathbb{E}^{d, d-1}\left(\begin{array}{c}
u_{0,1} \\
\vdots \\
u_{N, N} \\
v_{1,0} \\
\vdots \\
v_{N, N}
\end{array}\right) \\
& =\left(e_{1}(\xi) e_{1}(\eta) \ldots e_{N}(\xi) e_{N}(\eta)\right) \mathbb{E}^{d, d-1} \mathrm{u} .
\end{aligned}
$$

Note that $\mathbb{E}^{d, d-1}$ is the incidence matrix which also appeared in 10 and footnote 3 .

If we insert the expansion $\sqrt{32}$ in $\left(\tilde{\boldsymbol{u}}, \mathbb{K}^{-1} \boldsymbol{u}\right)$ we obtain 


$$
\left(\tilde{\boldsymbol{u}}, \mathbb{K}^{-1} \boldsymbol{u}\right)=\tilde{\mathrm{u}}^{T} \mathbb{M}_{\mathbb{K}^{-1}}^{(d-1)} \mathrm{u},
$$

with

$\mathbb{M}_{\mathbb{K}^{-1}}^{(d-1)}=$

$\iint_{\Omega}\left(\begin{array}{cc}h_{0}(\xi) e_{1}(\eta) & 0 \\ \vdots & \vdots \\ h_{N}(\xi) e_{N}(\eta) & 0 \\ 0 & e_{1}(\xi) h_{0}(\eta) \\ \vdots & \vdots \\ 0 & e_{N}(\xi) h_{N}(\eta)\end{array}\right) \mathbb{K}^{-1}\left(\begin{array}{ccccc}h_{0}(\xi) e_{1}(\eta) \ldots & h_{N}(\xi) e_{N}(\eta) & 0 & \ldots & 0 \\ 0 & \ldots & 0 & e_{1}(\xi) h_{0}(\eta) \ldots e_{N}(\xi) h_{N}(\eta)\end{array}\right) \mathrm{d} \Omega$

Note that pressure is expanded in the same basis as the divergence of the velocity field, (31) and (33), therefore we can write

$$
(\tilde{p}, \nabla \cdot \boldsymbol{u})=\tilde{\mathrm{p}}^{T} \mathbb{M}^{(d)} \mathbb{E}^{d, d-1} \mathrm{u},
$$

with

$$
\mathbb{M}^{(d)}=\iint_{\Omega}\left(\begin{array}{c}
e_{1}(\xi) e_{1}(\eta) \\
\vdots \\
e_{N}(\xi) e_{N}(\eta)
\end{array}\right)\left(e_{1}(\xi) e_{1}(\eta) \ldots e_{N}(\xi) e_{N}(\eta)\right) \mathrm{d} \Omega
$$

With (34) and 37) we can write 30 as

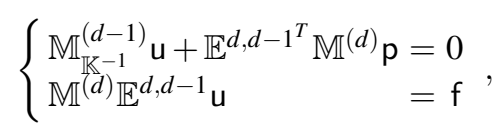

with

$$
\mathrm{f}=\iint_{\Omega}\left(\begin{array}{c}
e_{1}(\xi) e_{1}(\eta) \\
\vdots \\
e_{N}(\xi) e_{N}(\eta)
\end{array}\right) f(\xi, \eta) \mathrm{d} \Omega
$$

Comparison of (38) with (14) shows that the topological incidence matrices also appear in the finite element formulation and that the (weighted) mass matrices $\mathbb{M}_{\mathbb{K}^{-1}}^{(d-1)}$ and $\mathbb{M}^{(d)}$ once again play the role of the $\mathbb{H}$-matrices which connect solutions on dual grids.

In this section only the discretization on a single spectral element is discussed. Transformation of the domain $[-1,1]^{2}$ to more general domains will be discussed in Section 4. The use of multiple elements follows the general assembly procedure from finite element methods. Results of this approach are presented in Section 5 . 


\section{Transformation rules}

The basis functions used in the discretization of the different physical field quantities have only been introduced for the reference domain $\tilde{\Omega}=[-1,1]^{2}$. For these basis functions to be applicable in a different domain $\Omega$, it is fundamental to discuss how they transform under a mapping $\Phi:(\xi, \eta) \in \tilde{\Omega} \mapsto(x, y) \in \Omega \subset \mathbb{R}^{2}$. Within a finite element formulation this is particularly useful because the basis functions in the reference domain $\tilde{\Omega}$ can then be transformed to each of the elements $\Omega_{e}$, given a mapping $\Phi_{e}: \tilde{\Omega} \mapsto \Omega_{e}$.

Consider a smooth bijective map $\Phi:(\xi, \eta) \in \tilde{\Omega} \mapsto(x, y) \in \Omega$ such that

$$
x=\Phi^{x}(\xi, \eta) \quad \text { and } \quad y=\Phi^{y}(\xi, \eta),
$$

and the associated rank two Jacobian tensor J

$$
\mathrm{J}:=\left[\begin{array}{ll}
\frac{\partial \Phi^{x}}{\partial \xi} & \frac{\partial \Phi^{x}}{\partial \eta} \\
\frac{\partial \Phi^{y}}{\partial \xi} & \frac{\partial \Phi^{y}}{\partial \eta}
\end{array}\right] .
$$

The transformation of a scalar function $\varphi$ discretized by nodal values is given by

$$
\tilde{\varphi}(\xi, \eta)=(\varphi \circ \Phi)(\xi, \eta) \text { and } \quad \varphi(x, y)=\left(\tilde{\varphi} \circ \Phi^{-1}\right)(x, y),
$$

and of a scalar function $\rho$ discretized by surface integrals is given by

$$
\tilde{\rho}(\xi, \eta)=\operatorname{det} J(\rho \circ \Phi)(\xi, \eta) \quad \text { and } \quad \rho(x, y)=\frac{1}{\operatorname{det} J}\left(\tilde{\rho} \circ \Phi^{-1}\right)(x, y) .
$$

The transformation of vector fields $v$ discretized by line integrals is

$$
\tilde{\boldsymbol{v}}(\xi, \eta)=\mathrm{J}^{\top}(\boldsymbol{v} \circ \Phi)(\xi, \eta) \quad \text { and } \quad \boldsymbol{v}(x, y)=\left(\mathrm{J}^{\top}\right)^{-1}\left(\tilde{\boldsymbol{v}} \circ \Phi^{-1}\right)(x, y),
$$

and of vector fields $\boldsymbol{u}$ discretized by flux integrals is

$$
\tilde{\boldsymbol{u}}(\xi, \eta)=\operatorname{det} \mathrm{J}^{-1}(\boldsymbol{u} \circ \Phi)(\xi, \eta) \quad \text { and } \quad \boldsymbol{u}(x, y)=\frac{1}{\operatorname{det} \mathrm{J}} \mathrm{J}\left(\tilde{\boldsymbol{u}} \circ \Phi^{-1}\right)(x, y) .
$$

These transformations affect only the mass matrices and not the incidence matrices. This is fundamental to ensure the topological nature of the incidence matrices.

\section{Numerical results}

In this section three test cases are presented to illustrate the accuracy of the discretization scheme developed in this work. The first test case, 5.1 is an analytical 
solution taken from [68] to assess the convergence rates of the method. The second test case, 5.2. is the flow through a system of sand and shale blocks with highly heterogeneous permeability in the domain, see for more details [54]. The third test case, 5.3, is a highly anisotropic and heterogeneous permeability tensor in the domain, see for more details, [53].

\subsection{Manufactured solution}

We first test the method using the exact solution

$$
p_{\text {exact }}(x, y)=\sin (\pi x) \sin (\pi y),
$$

with the permeability tensor given by

$$
\mathbb{K}=\frac{1}{\left(x^{2}+y^{2}+\alpha\right)}\left(\begin{array}{cc}
10^{-3} x^{2}+y^{2}+\alpha & \left(10^{-3}-1\right) x y \\
\left(10^{-3}-1\right) x y & x^{2}+10^{-3} y^{2}+\alpha
\end{array}\right) .
$$

The mixed formulation (3) in the form of $(38)$ is then solved in the domain $(x, y) \in \Omega=[0,1]^{2}$ with the source term $f=-\nabla \cdot\left(\mathbb{K} \nabla p_{\text {exact }}\right)$ and the Dirichlet boundary condition $\left.p\right|_{\partial \Omega}=0$. A benchmark of this test case for $\alpha=0$ using multiple numerical schemes can be found in [68].

When $\alpha=0, \mathbb{K}$ is multi-valued at the origin which makes this test case a challenging one. To see this, we can first convert the Cartesian coordinates $(x, y)$ to polar coordinates $(r, \theta)$ by $x=r \cos \theta, y=r \sin \theta$. Then we have

$$
\left.\mathbb{K}\right|_{\alpha=0}=\left(\begin{array}{cc}
10^{-3} \cos ^{2} \theta+\sin ^{2} \theta & \left(10^{-3}-1\right) \cos \theta \sin \theta \\
\left(10^{-3}-1\right) \cos \theta \sin \theta & \cos ^{2} \theta+10^{-3} \sin ^{2} \theta
\end{array}\right) .
$$

It can be seen that we get different $\left.\mathbb{K}\right|_{\alpha=0}$ when we approach the origin along different angles, $\theta$. It must be noted that inverse of $\mathbb{K}$ does not exist at the origin. The inverse of the tensor term appears in 3.2.2. We use Gauss integration and thus the inverse term is not evaluated at the origin.

The meshes we use here are obtained by deforming the GLL meshes in the reference domain $(\xi, \eta) \in \Omega_{\mathrm{ref}}=[-1,1]^{2}$ with the mapping, $\Phi$, given as

$$
\left\{\begin{array}{l}
x=\frac{1}{2}+\frac{1}{2}(\xi+c \sin (\pi \xi) \sin (\pi \eta)) \\
y=\frac{1}{2}+\frac{1}{2}(\eta+c \sin (\pi \xi) \sin (\pi \eta))
\end{array}\right.
$$

where $c$ is the deformation coefficient. The two meshes, for $c=0.0$ and $c=0.3$, are shown in Figure 5.

The method is tested for $\alpha \in\{0,0.01\}$ and $c \in\{0,0.3\}$. 

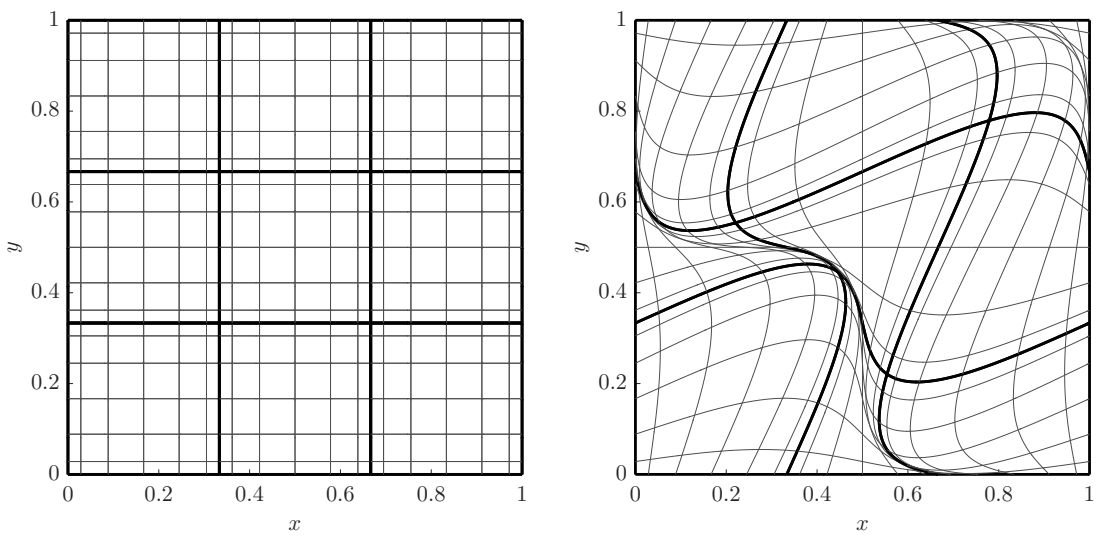

Fig. 5: Example meshes with $3 \times 3$ elements of polynomial degree $N=6$. Left: $c=0$ (orthogonal mesh). Right: $c=0.3$ (highly deformed mesh).

In Figure 6, the results for $\left\|\nabla \cdot \boldsymbol{u}_{h}-f_{h}\right\|_{L^{2}}$ are presented. They show that the relation $\nabla \cdot \boldsymbol{u}_{h}=f_{h}$ is conserved to machine precision even on a highly deformed and coarse mesh i.e. of $2 \times 2$ elements with $N=2$ and $c=0.3$.

When $\alpha=0.01, \mathbb{K}$ is no longer multi-valued at the origin. In this case the source term $f$ is smooth over the domain, see Figure 7 (bottom). For this smooth case, the method displays optimal convergence rates on both the orthogonal mesh and the deformed mesh, i.e. see Figure 8(bottom) and Figure 9 (bottom).

When $\alpha=0$, both the $h$-convergence rate and $p$-convergence rates are suboptimal, see Figure 8 (top) and Figure 9 (top). This is because $\mathbb{K}$ is multi-valued and therefore $f$ becomes singular at the origin when $\alpha=0$, see Figure 7(top left). 

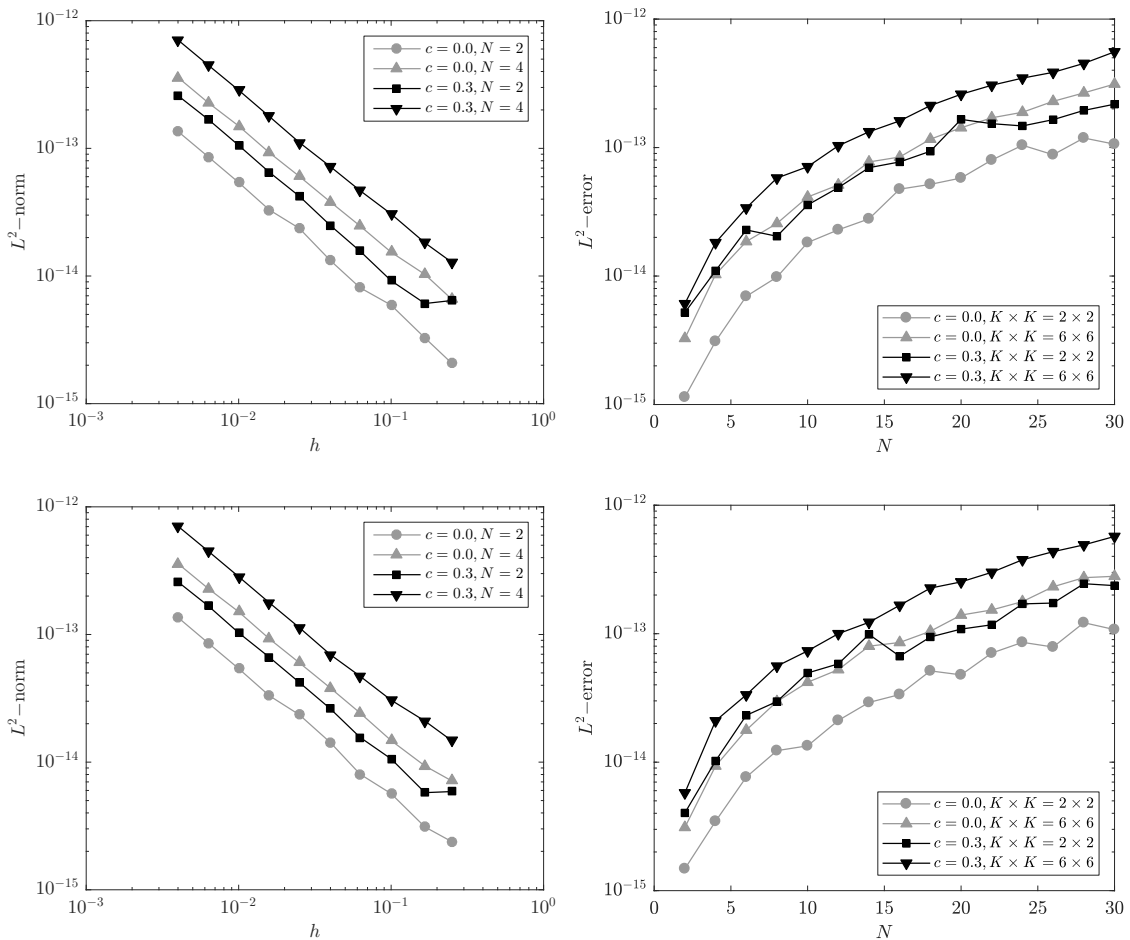

Fig. 6: The $L^{2}$-norm of $\left(\nabla \cdot \boldsymbol{u}_{h}-f_{h}\right)$. Left: $K \times K$ elements, $K=4, \ldots, 250$, and $N=$ 2,4. Right: $2 \times 2,6 \times 6$ elements, and $N=2, \ldots, 30$. Top: $\alpha=0$. Bottom: $\alpha=0.01$. 

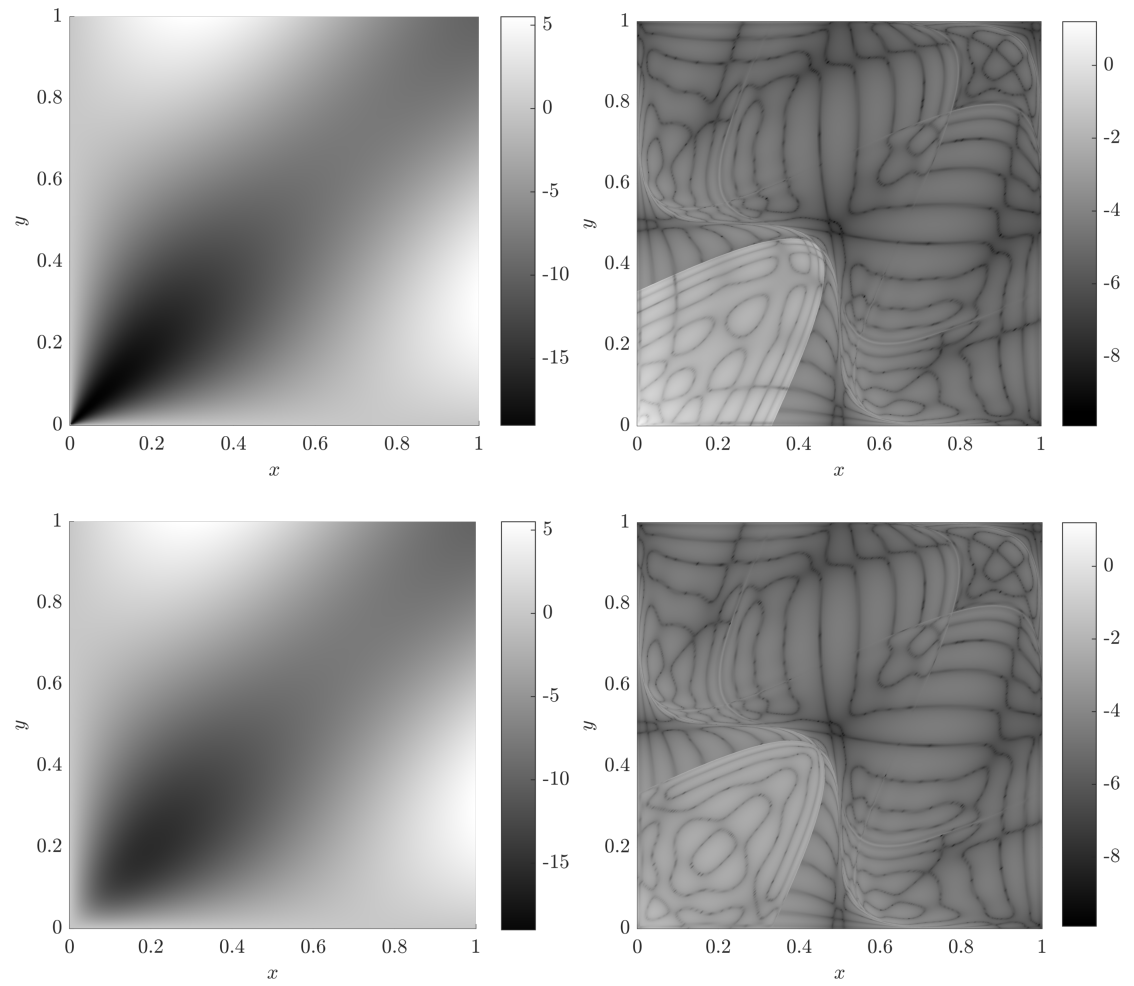

Fig. 7: Left: the source term $f$. Right: the $\log _{10}$ distribution of the projection error of $f_{h}$ for $3 \times 3$ elements, $N=10$ and $c=0.3$. Top: $\alpha=0$. Bottom: $\alpha=0.01$. 

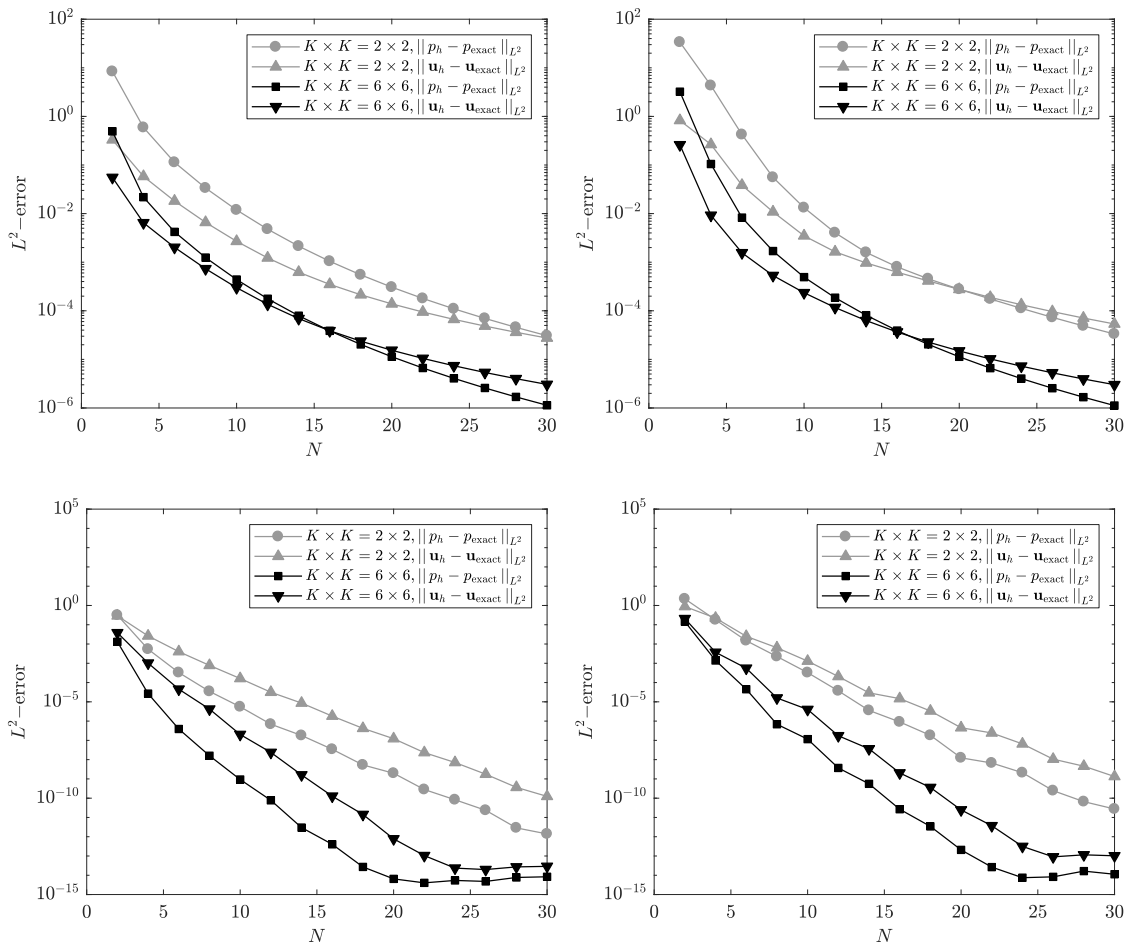

Fig. 8: The $p$-convergence for $2 \times 2,6 \times 6$ elements and $N=2, \ldots, 30$. Left: $c=0$. Right: $c=0.3$. Top: $\alpha=0$. Bottom: $\alpha=0.01$. 

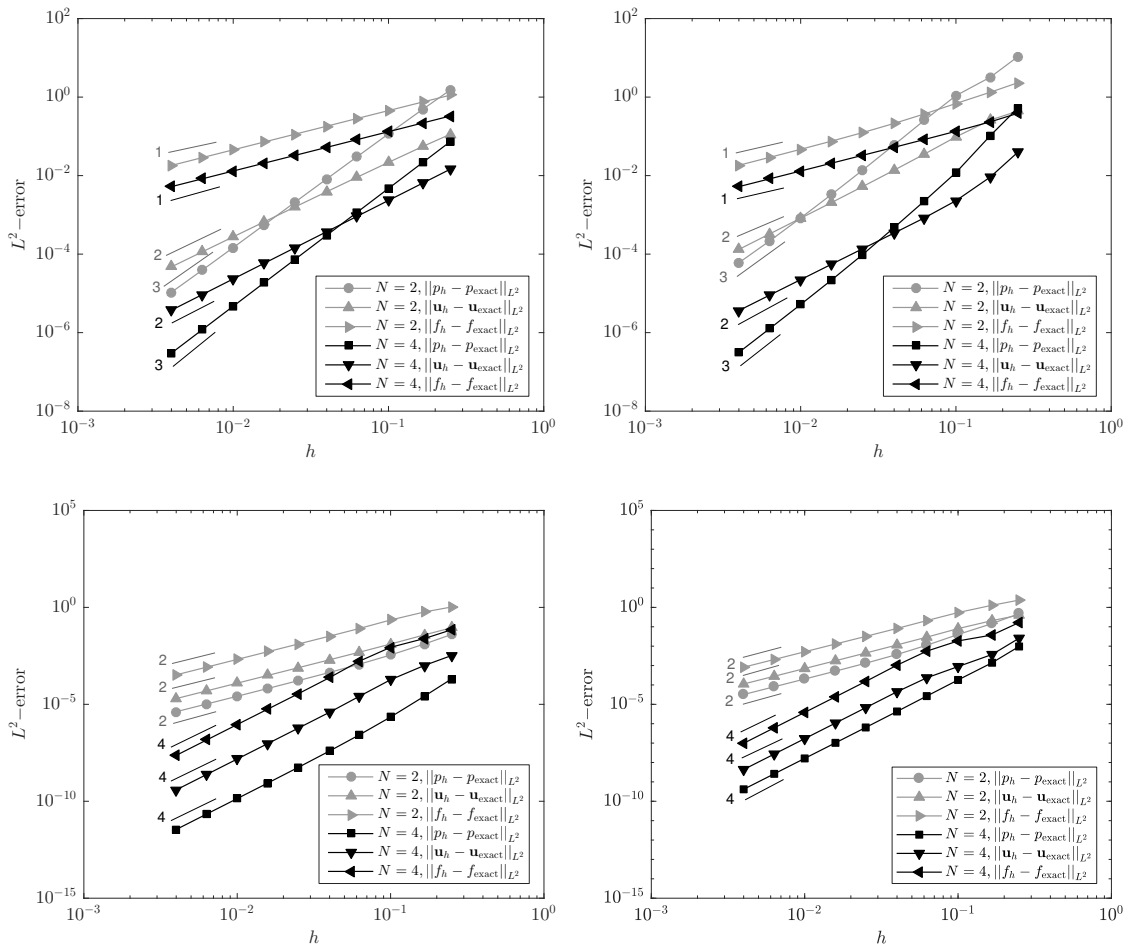

Fig. 9: The $h$-convergence of the $L^{2}$-error for $K \times K$ elements, $K=4, \ldots, 250$ and $N=2$, 4. Left: $c=0$. Right: $c=0.3$. Top: $\alpha=0$. Bottom: $\alpha=0.01$. 


\subsection{The Sand-Shale system}

This example is taken from [54,76, 78]. The domain is a $2 \mathrm{D}$ unit square, $\Omega=[0,1]^{2}$, with 80 shale blocks, $\Omega_{s}$, placed in the domain such that the total area fraction of shale blocks is $A_{\text {shale }}=20 \%$, as shown in Figure 10 .

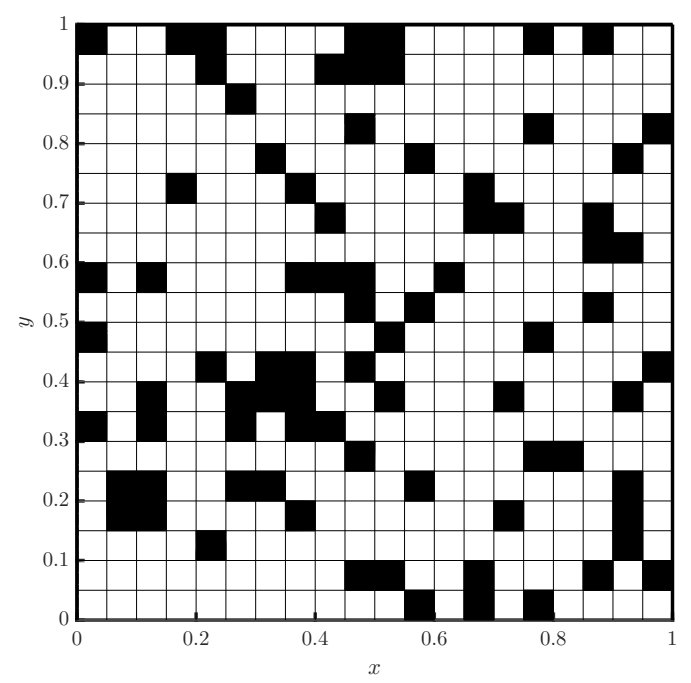

Fig. 10: The discretized domain for the sand-shale test case. Black blocks are shale blocks with $k=10^{-6}$. White blocks are sand blocks with $k=1$.

We solve the mixed formulation (38) with $f=0$ in this domain. The flux across the top and the bottom boundaries is $\boldsymbol{u} \cdot \boldsymbol{n}=0$. The flow is pressure driven with the pressure at the left boundary, $p=1$, and the pressure at the right boundary, $p=0$. The permeability in the domain is defined as $\mathbb{K}=k \mathbb{I}$, where $k$ is given by:

$$
k=\left\{\begin{array}{lll}
10^{-6} & \text { in } & \Omega_{s} \\
1 & \text { in } & \Omega \backslash \Omega_{s}
\end{array} .\right.
$$

For this test case an orthogonal uniform grid of $20 \times 20$ elements is used. The polynomial degree is varied to achieve convergence. Streamlines through the domain for $20 \times 20$ elements and polynomial degree $N=15$ are shown in Figure 11 . It can be seen that the streamlines do not pass through, but pass around the shale blocks of low permeability.

The $\left\|\nabla \cdot \boldsymbol{u}_{h}\right\|_{L^{2}}$ over the entire domain as a function of polynomial degree is shown in Figure 12. We observe that $\nabla \cdot \boldsymbol{u}_{h}=0$ is satisfied up to machine precision.

The net flux entering the domain (the same as the net flux leaving the domain) is given in Table 1 for varying polynomial degree. A reference value for this solution is given in [54] as 0.5205, and in [78] as 0.519269. In this work the maximum 


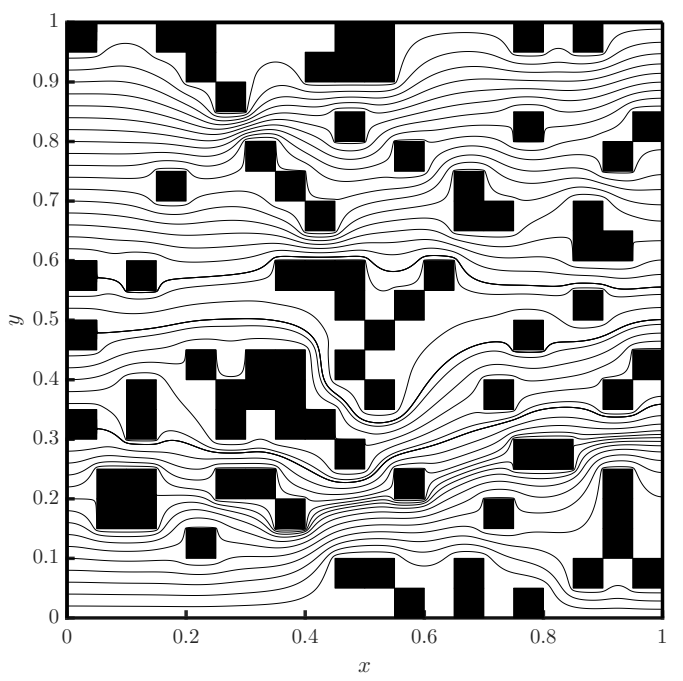

Fig. 11: Streamlines through the domain of sand-shale test case.

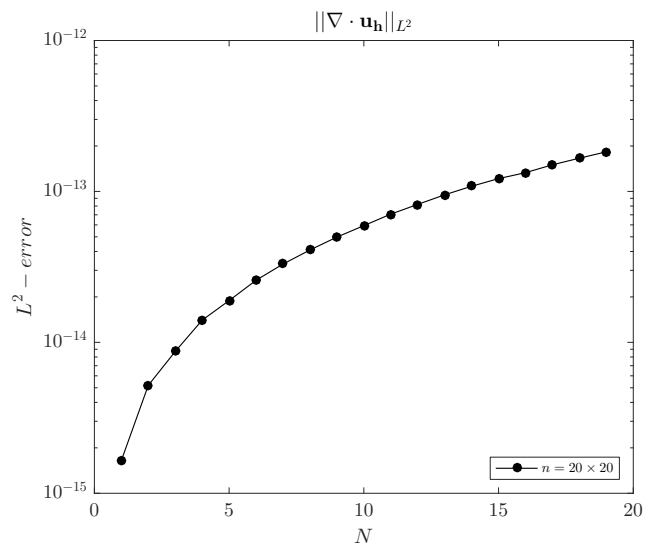

Fig. 12: The $L^{2}$-norm of $\nabla \cdot \boldsymbol{u}_{h}$ for $20 \times 20$ elements for a polynomial approximation of $N=1, \ldots, 19$.

resolution corresponds to $20 \times 20$ elements and a polynomial degree $N=19$, for which the net flux entering the domain is obtained as 0.52010 .

In Figure 13 we compare the net flux entering the sand-shale domain, calculated using the mixed and the direct formulation of equations, as a function of polynomial degree for different values of $k$ in the shale blocks. The data for these figures is given in Table 2 Note that the direct formulation converges from above towards the correct inflow flux, whereas the mixed formulation converges from below. 


\begin{tabular}{|c|c|c|}
\hline $\mathrm{N}$ & Net flux & of unknowns \\
\hline 1 & 0.49041 & 1240 \\
\hline 2 & 0.51247 & 4880 \\
\hline 3 & 0.51744 & 10920 \\
\hline 4 & 0.51863 & 19360 \\
\hline 5 & 0.51931 & 30200 \\
\hline 6 & 0.51957 & 43440 \\
\hline 7 & 0.51977 & 59080 \\
\hline 8 & 0.51985 & 77120 \\
\hline 9 & 0.51993 & 97560 \\
\hline 10 & 0.51997 & 120400 \\
\hline 11 & 0.52001 & 145640 \\
\hline 12 & 0.52003 & 173280 \\
\hline 13 & 0.52005 & 203320 \\
\hline 14 & 0.52007 & 235760 \\
\hline 15 & 0.52008 & 270600 \\
\hline 16 & 0.52009 & 307840 \\
\hline 17 & 0.52009 & 347480 \\
\hline 18 & 0.52010 & 389520 \\
\hline 19 & 0.52010 & 433960 \\
\hline
\end{tabular}

Table 1: Net flux through the left boundary of the sand-shale domain for $k=10^{-6}$, $20 \times 20$ elements, $N=1, \ldots, 19$.

\begin{tabular}{ccccccccc}
\hline $\mathrm{N}$ & \multicolumn{2}{c}{$k=10^{-1}$} & \multicolumn{2}{c}{$k=10^{-2}$} & \multicolumn{2}{c}{$k=10^{-3}$} & \multicolumn{2}{c}{$k=10^{-4}$} \\
& Mixed & Direct & Mixed & Direct & Mixed & Direct & Mixed & Direct \\
\hline 1 & 0.63805 & 0.74149 & 0.51384 & 0.69273 & 0.49296 & 0.68699 & 0.49066 & 0.68641 \\
2 & 0.66541 & 0.69316 & 0.54101 & 0.62399 & 0.51573 & 0.61572 & 0.51279 & 0.61488 \\
3 & 0.67131 & 0.68423 & 0.54906 & 0.60794 & 0.52121 & 0.59856 & 0.51782 & 0.59760 \\
4 & 0.67339 & 0.68139 & 0.55208 & 0.60113 & 0.52272 & 0.59099 & 0.51904 & 0.58995 \\
5 & 0.67450 & 0.68003 & 0.55436 & 0.59711 & 0.52371 & 0.58639 & 0.51975 & 0.58528 \\
6 & 0.67512 & 0.67926 & 0.55568 & 0.59439 & 0.52417 & 0.58320 & 0.52003 & 0.58203 \\
7 & 0.67555 & 0.67877 & 0.55690 & 0.59239 & 0.52459 & 0.58079 & 0.52026 & 0.57958 \\
8 & 0.67582 & 0.67844 & 0.55772 & 0.59085 & 0.52483 & 0.57890 & 0.52036 & 0.57765 \\
9 & 0.67604 & 0.67821 & 0.55852 & 0.58960 & 0.52508 & 0.57734 & 0.52046 & 0.57605 \\
10 & 0.67619 & 0.67803 & 0.55910 & 0.58857 & 0.52524 & 0.57603 & 0.52051 & 0.57471 \\
\hline
\end{tabular}

Table 2: Data of net flux through the left boundary of the sand-shale domain using mixed formulation and direct formulation for $20 \times 20$ elements, $N=1, \ldots, 10, k=$ $10^{-1}$ (top-left), $10^{-2}$ (top-right), $10^{-3}$ (bottom-left) and $10^{-4}$ (bottom-right). 

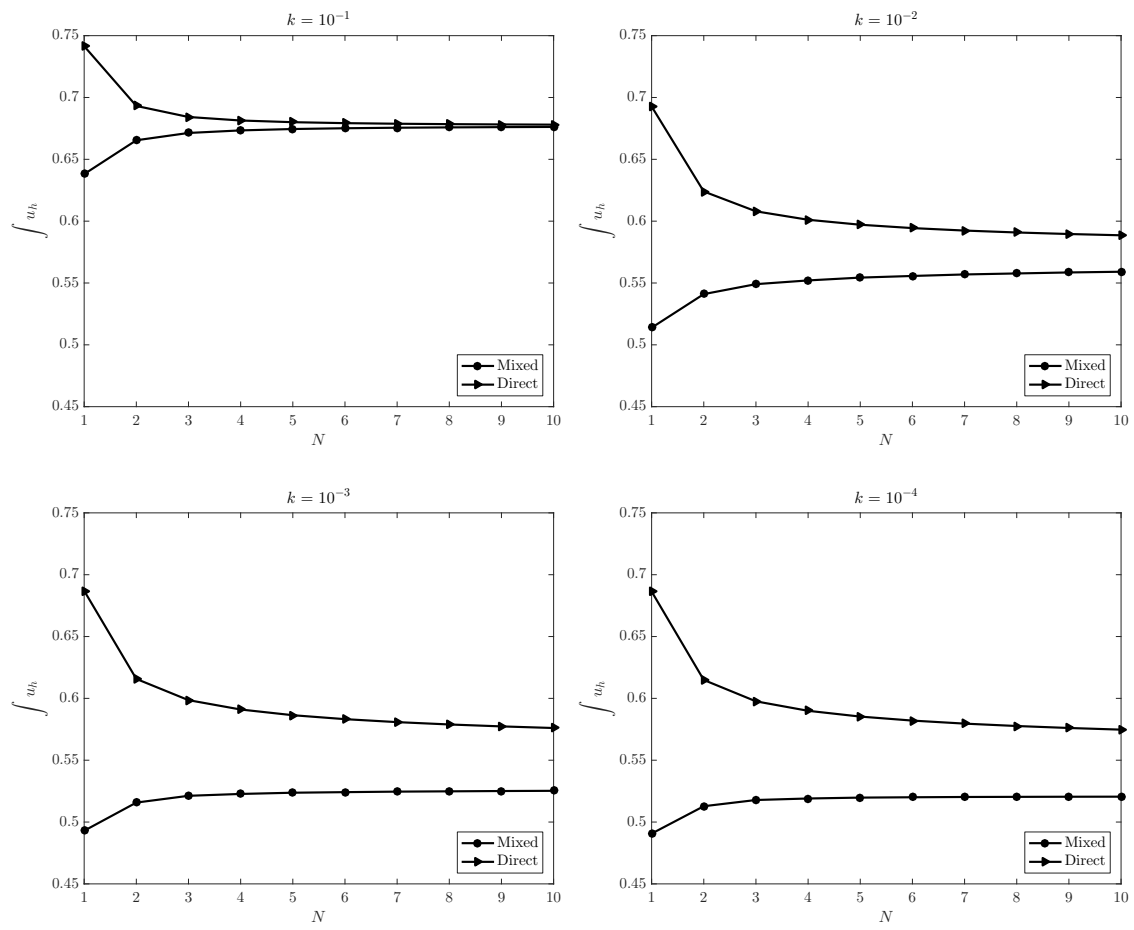

Fig. 13: Convergence of the net flux through the left boundary of the sand-shale domain using the mixed formulation and the direct formulation for $20 \times 20$ elements, $N=1, \ldots, 10$. Top left: $k=10^{-1}$. Top right: $k=10^{-2}$. Bottom left: $k=10^{-3}$. Bottom right: $k=10^{-4}$. 


\subsection{The Impermeable-Streak system}

The next example is from [53, 76, 78]. The physical domain is a 2D unit square, $\Omega=[0,1]^{2}$. The domain is divided into three different regions, $\Omega_{1}, \Omega_{2}$, and $\Omega_{3}$,
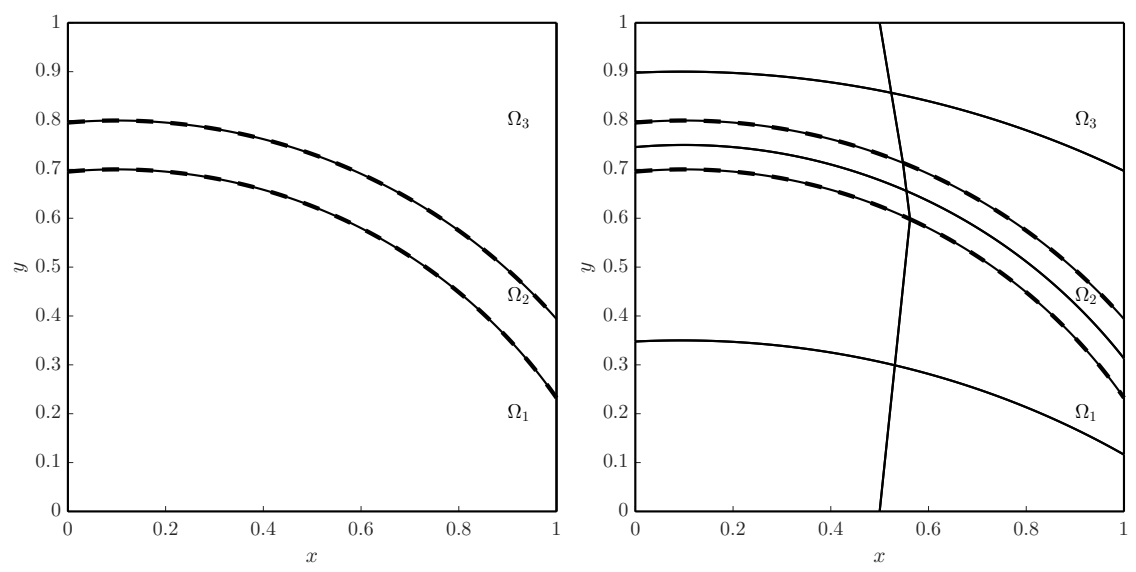

Fig. 14: Three regions of the domain for the impermeable streak test case. The regions are separated by the dashed lines. The solid lines indicate the element boundaries. Left: $1 \times 1$ element in each region. Right: $2 \times 2$ elements in each region.

as shown in Figure 14 (left). For calculations, each region is further divided into $K \times K$ elements. Therefore, the total number of elements in the domain is given by $K \times K \times 3$. In Figure 14 (right) we show the domain with each region divided into $2 \times 2$ elements.

The mixed formulation 38 is solved, with $f=0$ and mixed boundary conditions, such that at the top and the bottom boundaries the net flux $\boldsymbol{u} \cdot \boldsymbol{n}=0$, and at the left and the right boundaries, $p=1$ and $p=0$, respectively. Permeability in $\Omega_{1}$ and $\Omega_{3}$ is given by $\mathbb{K}=\mathbb{I}$. $\Omega_{2}$ has a low permeability and defined such that the component parallel to the local streak orientation is $k_{\|}=10^{-1}$, and the component perpendicular to the local streak orientation is $k_{\perp}=10^{-3}$. The analytical expression for the permeability in terms of Cartesian coordinates is given in [76] as,

$$
\begin{aligned}
& K_{x x}=\frac{k_{\|}(y+0.4)^{2}+k_{\perp}(x-0.1)^{2}}{(x-0.1)^{2}+(y+0.4)^{2}}, \\
& K_{x y}=\frac{-\left(k_{\|}-k_{\perp}\right)(x-0.1)(y+0.4)}{(x-0.1)^{2}+(y+0.4)^{2}}, \\
& K_{y y}=\frac{k_{\|}(x-0.1)^{2}+k_{\perp}(y+0.4)^{2}}{(x-0.1)^{2}+(y+0.4)^{2}} .
\end{aligned}
$$




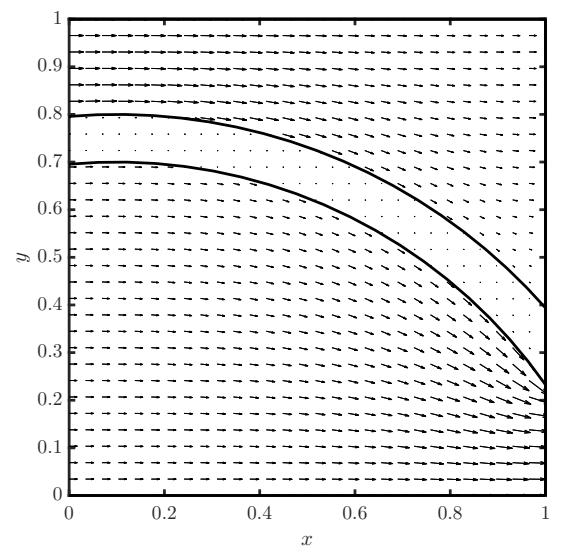

Fig. 15: Velocity vectors through the domain of permeability streak test case for $12 \times 12$ elements, $N=15$.

The flow field in the domain is shown in Figure 15. The magnitude of velocity in $\Omega_{2}$ is small due to low values of the permeability tensor in this region. The velocity vectors bend in the direction of the permeability streak $\Omega_{2}$. The $L^{2}$-norm of $\nabla \cdot \boldsymbol{u}$ over the entire domain as a function of polynomial degree, $N$, is shown in Figure 16 . We can see that the flow field is divergence free up to machine precision because $f=0$.

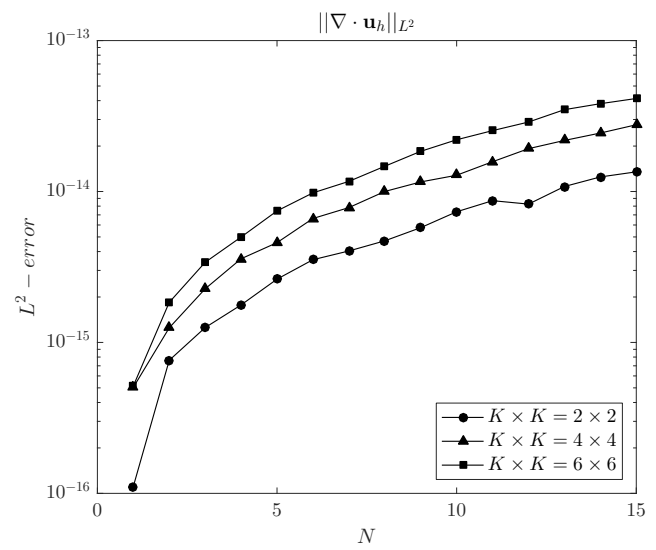

Fig. 16: The $L^{2}$-norm of $\nabla \cdot \boldsymbol{u}_{h}$ for $K \times K$ elements, $K=2,4,6, N=1, \ldots, 15$.

The net flux through the system for varying number of elements and polynomial degree is given in Table 3 . In this work the finest resolution corresponds to $12 \times 12 \times$ 3 elements and $N=15$. For this case the net influx at the left boundary is 0.75668 . 
The net influx and outflux from the region $\Omega_{1}, \Omega_{2}$ and $\Omega_{3}$ is given in Tables 4,5 , and 6 , respectively. The net influx for $\Omega_{1}$ is larger than the net outflux. And the net outflux for $\Omega_{2}$ and $\Omega_{3}$ is larger than the net influx.

\begin{tabular}{lcccccc}
\hline $\mathrm{N}$ & \multicolumn{5}{c}{ Elements Division $(K \times K)$} \\
& $4 \times 4$ & $6 \times 6$ & $8 \times 8$ & $10 \times 10$ & $12 \times 12$ \\
\hline 1 & 0.74689 & 0.74908 & 0.75061 & 0.75169 & 0.75247 \\
2 & 0.75268 & 0.75407 & 0.75479 & 0.75522 & 0.75550 \\
3 & 0.75479 & 0.75548 & 0.75582 & 0.75602 & 0.75615 \\
4 & 0.75561 & 0.75600 & 0.75620 & 0.75631 & 0.75639 \\
5 & 0.75600 & 0.75625 & 0.75638 & 0.75645 & 0.75650 \\
6 & 0.75621 & 0.75639 & 0.75648 & 0.75653 & 0.75657 \\
7 & 0.75635 & 0.75648 & 0.75654 & 0.75658 & 0.75660 \\
8 & 0.75643 & 0.75653 & 0.75658 & 0.75661 & 0.75663 \\
9 & 0.75649 & 0.75657 & 0.75661 & 0.75663 & 0.75665 \\
10 & 0.75654 & 0.75660 & 0.75663 & 0.75665 & 0.75666 \\
11 & 0.75657 & 0.75662 & 0.75664 & 0.75666 & 0.75667 \\
12 & 0.75659 & 0.75663 & 0.75665 & 0.75666 & 0.75667 \\
13 & 0.75661 & 0.75664 & 0.75666 & 0.75667 & 0.75668 \\
14 & 0.75662 & 0.75665 & 0.75667 & 0.75668 & 0.75668 \\
15 & 0.75663 & 0.75666 & 0.75667 & 0.75668 & 0.75668 \\
\hline
\end{tabular}

Table 3: Net flux through the left boundary of the permeability streak test case domain for $K \times K$ elements, $K=4,6,8,10,12$ and $N=1, \ldots, 15$.

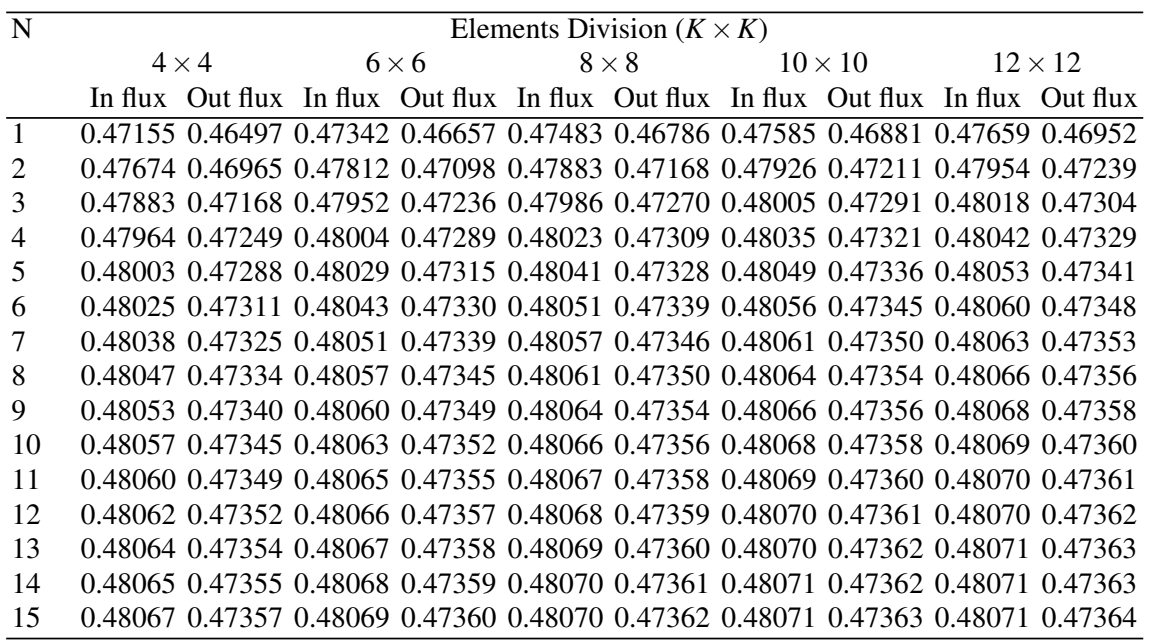

Table 4: Net flux through the left boundary of the region $\Omega_{1}$ for $K \times K$ elements, $K=4,6,8,10,12$ and $N=1, \ldots, 15$. 


\begin{tabular}{|c|c|c|c|c|c|c|c|c|}
\hline \multirow[t]{3}{*}{$\mathrm{N}$} & \multicolumn{8}{|c|}{ Elements Division $(K \times K)$} \\
\hline & $4 \times 4$ & $6 \times 6$ & \multicolumn{2}{|c|}{$8 \times 8$} & \multicolumn{2}{|c|}{$10 \times 10$} & \multicolumn{2}{|c|}{$12 \times 12$} \\
\hline & In flux Out flux & In flux Out flux & In flux & Out flux & In flux & Out flux & In flux & Out flux \\
\hline 1 & 0.009300 .01080 & 0.009310 .01106 & 0.00931 & 0.01119 & 0.00932 & 0.01130 & 0.00932 & 0.01130 \\
\hline 2 & 0.009320 .0 & 0.009330. & 0.00933 & 0.01139 & 0.00933 & & $0.0 c$ & 0.01140 \\
\hline 3 & 330.01 & 0.009330 .01140 & 0.00933 & 0.01140 & 0.00933 & 0.01 & 0.00 & 0.01139 \\
\hline 4 & 0.00 & 330.0 & 0.00 & 0.0114 & 0.00934 & 0.0 & 0.00934 & 0.01139 \\
\hline 5 & 0.00 & 0 & 0.0 & 0.0 & 0.00934 & $0 .($ & 0.00 & 0.01138 \\
\hline 6 & 0.009340 .0 & 0.009340 .0113 & 0.00934 & 0.0 & 0.00934 & 0 & 0.00 & 0.01137 \\
\hline & 40. & 0.009340 .0 & 934 & 0.0 & 0.00934 & 0 & 0 . & 136 \\
\hline & 40. & 40. & 0 & & 0.0 & & & 136 \\
\hline & +0 & +0 & & & & & & 35 \\
\hline 10 & 0.0 & 40. & & & 0.0 & & & 135 \\
\hline 11 & $0.1+3$ & 0. & & & 0.0 & & & 135 \\
\hline 12 & 0.00 & 0.0 & 0. & & 0.0 & & & 134 \\
\hline 13 & 0.009340 .0 & 0.009340 .01135 & 0.00934 & 0.01135 & 0.00934 & 0.01 & 0.00934 & 0.01134 \\
\hline 14 & 40.0 & 0.009340 .01135 & 0.00934 & 0.011 & 0.00934 & 0.0 & 0.00934 & 0.01134 \\
\hline 15 & 0.009340 .01135 & 0.009340 .01135 & 0.00934 & 0.01134 & 0.00934 & 0.01134 & 0.00934 & 0.01134 \\
\hline
\end{tabular}

Table 5: Net flux through the left boundary of the region $\Omega_{2}$ for $K \times K$ elements, $K=4,6,8,10,12$ and $N=1, \ldots, 15$.

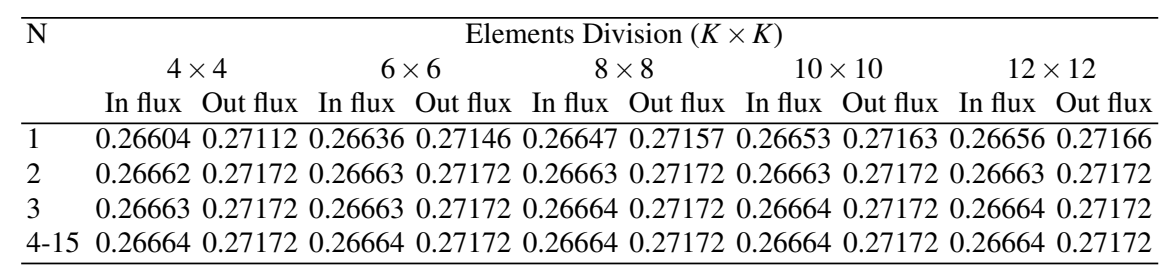

Table 6: Net flux through the left boundary of the region $\Omega_{3}$ for $K \times K$ elements, $K=4,6,8,10,12$ and $N=1, \ldots, 15$.

\section{Future Work}

In the above sections, mixed and direct formulations of mimetic spectral element method are discussed. The next step is to explore this framework in the direction of hybrid formulations [20, 35, 43]. Additionally, the focus will be on developing multiscale methods [118], using these formulations, for reservoir modelling applications.

\section{References}

[1] J. E. Aarnes, S. Krogstad, And K.-A. Lie, Multiscale mixed/mimetic methods on corner-point grids, Computational Geosciences, 12 (2008), pp. 297-315. 
[2] I. AaVATSMark, An Introduction to multipoint flux approximations for quadrilateral grids, Computational Geosciences, 6 (2002), pp. 405-432.

[3] , Interpretation of a two-point flux stencil for skew parallelogram grids, Computational Geosciences, 11 (2007), pp. 199-206.

[4] I. Aavatsmark, T. Barkve, O. Bøe, And T. Mannseth, Discretization on unstructured grids for inhomogeneous, anisotropic media. Part I: derivation of the methods, SIAM Journal on Scientific Computing, 19 (1998), pp. 1700-1716.

[5] — Discretization on unstructured grids for inhomogeneous, anisotropic media. Part II: discussion and numerical results, SIAM Journal on Scientific Computing, 19 (1998), pp. 1717-1736.

[6] F. O. ALPAK, A mimetic finite volume discretization operator for reservoir simulation, in SPE Reservoir Simulation Symposium, Society of Petroleum Engineers, 2007.

[7] F. O. AlPAK, A mimetic finite volume discretization method for reservoir simulation, SPE Journal, 15 (2010), pp. 436-453.

[8] D. N. ARnold, D. Boffi, AND R. S. FALK, Quadrilateral H(div) Finite Elements, SIAM Journal on Numerical Analysis, 42 (2005), pp. 2429-2451.

[9] D. N. ARNOLD, R. S. FALK, AND R. WinTHER, Finite element exterior calculus, homological techniques, and applications, Acta Numerica, 15 (2006), pp. 1-155.

[10] — Finite element exterior calculus: from Hodge theory to numerical stability, Bulletin of the American Mathematical Society, 47 (2010), pp. 281354.

[11] K. AzIZ, Reservoir simulation grids: opportunities and problems, Journal of Petroleum Technology, 45 (1993), pp. 658-663.

[12] I. BABUSKA AND M. SURI, On locking and robustness in the finite element method, SIAM Journal on Numerical Analysis, 29 (1992), pp. 1261-1293.

[13] P. Bastian, O. IpPisch, And S. Marnach, Benchmark 3D: A Mimetic Finite Difference Method, in Finite Volumes for Complex Applications VI: Problems and Perspectives, Springer, Berlin, Heidelberg, 2011, pp. 961-968.

[14] W. BAUER AND F. GAY-BALMAZ, Variational integrators for anelastic and pseudo-incompressible flows, (2017).

[15] T. L. BERgman AND F. P. InCROPERA, Fundamentals of heat and mass transfer., Wiley, 2011.

[16] P. B. BocheV AND M. Gerritsma, A spectral mimetic least-squares method, Computers and Mathematics with Applications, 68 (2014), pp. 1480-1502.

[17] P. B. Bochev AND M. D. GunZBurger, Least-Squares Finite Element Methods, Springer series in Applied Mathematical Sciences, Springer-Verlag, 2009.

[18] P. B. Bochev And J. M. Hyman, Principles of mimetic discretizations of differential operators, IMA Volumes In Mathematics and its Applications, 142 (2006), p. 89. 
[19] P. B. Bochev AND D. RIDZAL, Rehabilitation of the Lowest-Order Raviart-Thomas Element on Quadrilateral Grids, SIAM Journal on Numerical Analysis, 47 (2008), pp. 487-507.

[20] D. Boffi, F. BRezzI, AND M. Fortin, Mixed Finite Element Methods and Applications, Springer Series in Computational Mathematics, SpringerVerlag, 2013.

[21] D. BOFFI AND L. GASTALDI, Some remarks on quadrilateral mixed finite elements, Computer and Structures, 87 (2009), pp. 751-757.

[22] J. Bonelle, E. Burman, P. Cantin, And A. ERn, A vertex-based scheme on polyhedral meshes for advection-reaction equations with submesh stabilization, hal-01285957, (2016), pp. 1-20.

[23] J. Bonelle, D. A. Di Pietro, AND A. ERn, Low-order reconstruction operators on polyhedral meshes: application to compatible discrete operator schemes, Computer Aided Geometric Design, 35-36 (2015), pp. 27-41.

[24] J. Bonelle AND A. ERn, Analysis of Compatible Discrete Operator schemes for elliptic problems on polyhedral meshes, ESAIM: Mathematical Modelling and Numerical Analysis, 48 (2014), pp. 553-581.

[25] _ Analysis of compatible discrete operator schemes for the Stokes equations on polyhedral meshes, IMA Journal of Numerical Analysis, 35 (2015), pp. 1672-1697.

[26] A. Boss AVIT, Computational electromagnetism and geometry: (1) Network equations, Journal of the Japan Society of Applied Electromagnetics, 7 (1999), pp. 150-159.

[27] _ Computational electromagnetism and geometry: (2) Network constitutive laws, Journal of the Japan Society of Applied Electromagnetics, 7 (1999), pp. 294-301.

[28] - Computational electromagnetism and geometry: (3) Convergence, Journal of the Japan Society of Applied Electromagnetics, 7 (1999), pp. 401408.

[29] — Computational electromagnetism and geometry: (4) From degrees of freedom to fields, Journal of the Japan Society of Applied Electromagnetics, 8 (2000), pp. 102-109.

[30] _ Computational electromagnetism and geometry: (5) The "Galerkin Hodge", Journal of the Japan Society of Applied Electromagnetics, 8 (2000), pp. 203-209.

[31] M. Bouman, A. Palha, J. Kreeft, and M. Gerritsma, a Conservative Spectral Element Method for Curvilinear Domains, in Spectral and High Order Methods for Partial Differential Equations, vol. 76 of Lecture Notes in Computational Science and Engineering, Springer, 2011, pp. 111-119.

[32] F. BREZZI AND A. BUfFA, Innovative mimetic discretizations for electromagnetic problems, Journal of Computational and Applied Mathematics, 234 (2010), pp. 1980-1987.

[33] F. BrezZI, A. Buffa, AND K. LiPNIKOV, Mimetic finite differences for elliptic problems, Mathematical Modelling and Numerical Analysis, 43 (2009), pp. 277-296. 
[34] F. BRezZI, R. S. FAlK, AND L. Donatella Marini, Basic principles of mixed Virtual Element Methods, ESAIM: Mathematical Modelling and Numerical Analysis, 48 (2014), pp. 1227-1240.

[35] F. BREzZI AND M. Fortin, Mixed and Hybrid Finite Element Methods, vol. 15 of Springer Series in Computational Mathematics, Springer, 1991.

[36] F. BREzzI, K. LipNikov, AND M. ShashKov, Convergence of mimetic finite difference method for diffusion problems on polyhedral meshes with curved faces, Mathematical Models and Methods in Applied Sciences, 16 (2006), pp. 275-297.

[37] F. BrezZi, K. Lipnikov, M. Shashrov, And V. Simoncini, A new discretization methodology for diffusion problems on generalized polyhedral meshes, Computer Methods in Applied Mechanics and Engineering, 196 (2007), pp. 3682-3692.

[38] F. BREZZI, K. LiPNIKov, AND V. SimonCINI, A family of mimetic finite difference methods on polygonal and polyhedral meshes, Mathematical Models and Methods in Applied Sciences, 15 (2005), pp. 1533-1551.

[39] C. Budd And M. PiggotT, Geometric Integration and its Applications, in Handbook of Numerical Analysis, vol. 11, North-Holland, 2003, pp. 35-139.

[40] A. Buffa, D. DE FAlCO, AND G. SANGALli, Isogeometric analysis: new stable elements for the Stokes equation, International Journal for Numerical Methods in Fluids, (2011).

[41] C. Canuto, M. Y. Hussaini, A. Quarteroni, And T. A. Zang, Spectral Methods in Fluid Dynamics, Springer Verlag, 1988.

[42] S. H. Christiansen, H. Z. Munthe-KaAs, And B. Owren, Topics in structure-preserving discretization, Acta Numerica, 20 (2011), pp. 1-119.

[43] B. Cock BURn, Static Condensation, Hybridization, and the Devising of the HDG Methods, Springer International Publishing, 2016, pp. 129-177.

[44] L. B. DA Veiga, F. Brezzi, L. D. Marini, And A. Russo, The Hitchhiker's Guide to the Virtual Element Method, Mathematical Models and Methods in Applied Sciences, 24 (2014), pp. 1541-1573.

[45] L. B. Da Veiga, F. Brezzi, L. D. Marini, and A. Russo, $H$ (div) and $H$ (curl) conforming virtual element methods, Numerische Mathematik, (2015), pp. 1-30.

[46] L. B. Da Veiga, F. Brezzi, L. D. Marini, and A. Russo, Virtual Element Method for general second-order elliptic problems on polygonal meshes, Mathematical Models and Methods in Applied Sciences, 26 (2016), pp. 729-750.

[47] L. B. DA Veiga, K. Lipnikov, and G. Manzini, The Mimetic Finite Difference Method for Elliptic Problems, Springer International Publishing, 2014.

[48] L. B. DA Veiga, C. Lovadina, And G. VACCA, Divergence free Virtual Elements for the Stokes problem on polygonal meshes, arXiv:1510.01655v1, (2015).

[49] M. Desbrun, A. N. Hirani, M. Leok, And J. E. Marsden, Discrete exterior calculus, arXiv:math/0508341v2, (2005). 
[50] D. A. Di Pietro AND A. ERn, Hybrid high-order methods for variablediffusion problems on general meshes, Comptes Rendus Mathematique, 353 (2015), pp. 31-34.

[51] D. A. Di Pietro, A. ERn, AND S. Lemaire, An arbitrary-order and compact-stencil discretization of diffusion on general meshes based on local reconstruction operators, Computational Methods in Applied Mathematics, 14 (2014).

[52] J. DodZIuk, Finite difference approach to the Hodge theory of harmonic functions, American Journal of Mathematics, 98 (1976), pp. 79-104.

[53] L. J. Durlofsky, A Triangle Based Mixed Finite Element Finite Volume Technique for Modeling Two Phase Flow through Porous Media, Journal of Computational Physics, 105 (1993), pp. 252-266.

[54] _ Accuracy of mixed and control volume finite element approximations to Darcy velocity and related quantities, Water Resources Research, 30 (1994), pp. 965-973.

[55] A. Dziubek, G. Guidoboni, A. Harris, A. N. Hirani, E. Rusjan, AND W. THISTLETON, Effect of ocular shape and vascular geometry on retinal hemodynamics: a computational model, Biomechanics and Modeling in Mechanobiology, 15 (2016), pp. 893-907.

[56] M. G. EDWARDS, Unstructured, control-volume distributed, full-tensor finitvolume schemes with flow based grids, Computational Geosciences, 6 (2002), pp. 433-452.

[57] M. G. EdWARds AND C. F. Rogers, Finite volume discretization with imposed flux continuity for the general tensor pressure equation, Computational Geosciences, 2 (1998), pp. 259-290.

[58] S. Elcott, Y. Tong, E. Kanso, P. Schröder, And M. Desbrun, Stable, circulation-preserving, simplicial fluids, ACM TRansactions on Graphics, 26 (2007).

[59] J. A. Evans And T. J. Hughes, Isogeometric divergence-conforming Bsplines for the unsteady Navier-Stokes equations, Journal of Computational Physics, 241 (2013), pp. 141-167.

[60] P. A. Forsyth, A control-volume, finite-element method for local mesh refinement in thermal reservoir simulation, SPE Reservoir Engineering, 5 (1990), pp. 561-566.

[61] M. GerRitsma, Edge Functions for Spectral Element Methods, in Spectral and High Order Methods for Partial Differential Equations, vol. 76 of Lecture Notes in Computational Science and Engineering, Springer, 2011, pp. 199207.

[62] M. GerRitsma AND P. B. BocheV, A spectral mimetic least-squares method for the Stokes equations with no-slip boundary condition, Computers and Mathematics with Applications, 71 (2016), pp. 2285-2300.

[63] M. Gerritsma, M. Bouman, And A. Palha, Least-Squares Spectral Element Method on a Staggered Grid, in Large-Scale Scientific Computing, vol. 5910 of Lecture Notes in Computer Science, Springer, 2010, pp. 653661. 
[64] M. Gerritsma, R. Hiemstra, J. Kreeft, A. Palha, P. P. Rebelo, And D. Toshniwal, The Geometric Basis of Numerical Methods, in Spectral and High Order Methods for Partial Differential Equations, vol. 95 of Lecture Notes in Computational Science and Engineering, Springer, 2013, pp. 17-35.

[65] D. Gunasekera, J. Cox, And P. Lindsey, The Generation and Application of K-Orthogonal Grid Systems, in SPE Reservoir Simulation Symposium, Society of Petroleum Engineers, 1997, pp. 199-214.

[66] E. Hairer, C. LubiCH, And G. WAnner, Geometric Numerical Integration, Springer, 2006.

[67] Z. E. Heinemann, C. W. Brand, M. Munka, And Y. M. Chen, Modeling reservoir geometry with irregular grids, SPE Reservoir Engineering, 6 (1991), pp. 225-232.

[68] R. HERBIN AND F. HUBERT, Benchmark on discretization schemes for anisotropic diffusion problems on general grids, in Finite Volumes for Complex Applications V: Problems and Perspectives, Wiley, 2008, pp. 659-692.

[69] F. HERMELINE, A finite volume method for the approximation of diffusion operators on distorted meshes, Journal of Computational Physics, 160 (2000), pp. 481-499.

[70] R. Hiemstra, D. Toshniwal, R. Huijsmans, and M. Gerritsma, High order geometric methods with exact conservation properties, Journal of Computational Physics, 257 (2014), pp. 1444-1471.

[71] R. Hiptmair, PIER, in Geometric Methods for Computational Electromagnetics, vol. 42, EMW Publishing, 2001, pp. 271-299.

[72] A. Hirani, Discrete Exterior Calculus, PhD thesis, California Institute of Technology, 2003.

[73] A. N. HiRAni, K. B. NAKshatrala, AND J. H. Chaudhry, Numerical method for Darcy flow derived using discrete exterior calculus, International Journal for Computational Methods in Engineering Science and Mechanics, 16 (2015), pp. 151-169.

[74] J. M. Hyman, J. Morel, M. Shashkov, and S. Steinberg, Mimetic finite difference methods for diffusion equations, Computational Geosciences, 6 (2002), pp. 333-352.

[75] J. M. HyMAn AND J. C. Scovel, Deriving mimetic difference approximations to differential operators using algebraic topology, tech. rep., Los Alamos National Laboratory, 1990.

[76] J. M. HYMAN, M. SHASHKOV, AND S. STEINBERG, The numerical solution of diffusion problems in strongly heterogeous non-isotropic materials, Journal of Computational Physics, 132 (1997), pp. 130-148.

[77] J. M. HYMAN AND S. STEINBERG, The convergence of mimetic methods for rough grids, Computers and Mathematics with applications, 47 (2004), pp. 1565-1610.

[78] E. Kikinzon, Y. Kuznetsov, K. Lipnikov, And M. Shashkov, Approximate static condensation algorithm for solving multi-material diffusion 
problems on meshes non-aligned with material interfaces, Journal of Computational Physics, (2017).

[79] S. Kouranbaeva AND S. ShKoller, A variational approach to secondorder multisymplectic field theory, Journal of Geometry and Physics, 35 (2000), pp. 333-366.

[80] M. KRAUS AND O. MAJ, Variational integrators for nonvariational partial differential equations, Physica D: Nonlinear Phenomena, 310 (2015), pp. 3771.

[81] J. Kreeft And M. Gerritsma, Mixed mimetic spectral element method for Stokes flow: A pointwise divergence-free solution, Journal of Computational Physics, 240 (2013), pp. 284-309.

[82] J. Kreeft, A. Palha, AND M. Gerritsma, Mimetic framework on curvilinear quadrilaterals of arbitrary order, arXiv:1111.4304, (2011), p. 69.

[83] K. Lie, S. Krogstad, I. S. LigaArden, J. R. Natvig, H. M. Nilsen, AND B. SKAFLESTAD, Open-source MATLAB implementation of consistent discretisations on complex grids, Computational Geosciences, 16 (2012), pp. 297-322.

[84] G. Manzini And M. Putti, Mesh locking effects in the finite volume solution of 2-D anisotropic diffusion equations, Journal of Computational Physics, 220 (2007), pp. 751-771.

[85] J. E. MARSDEN AND M. WeSt, Discrete mechanics and variational integrators, Acta Numerica 2001, 10 (2003), pp. 357-514.

[86] P. Mullen, K. Crane, D. Pavlov, Y. Tong, and M. Desbrun, Energy-preserving integrators for fluid animation, ACM Transactions on Graphics, 28 (2009).

[87] S. P. Neuman, Theoretical derivation of Darcy's law, Acta Mechanica, 25 (1977), pp. 153-170.

[88] R. NicOlaIDES, Discrete Discretization of planar div-curl problems, SIAM Journal on Numerical Analysis, 29 (1992), pp. 32-56.

[89] H. M. Nilsen, J. R. NATVIG, AND K.-A. LiE, Accurate Modeling of Faults by Multipoint, Mimetic, and Mixed Methods, SPE Journal, (2012), pp. pp. 568-579.

[90] C. L. PAlagi AND K. AzIZ, Use of Voronoi grid in reservoir simulation, SPE Advanced Technology Series, 2 (1994), pp. 69-77.

[91] A. Palha ANd M. GerRITSma, Mimetic Least-Squares Spectral/hp Finite Element Method for the Poisson Equation, in Large-Scale Scientific Computing, vol. 5910 of Lecture Notes in Computer Science, Springer, 2010, pp. 662-670.

[92] A. Palha And M. Gerritsma, Spectral Element Approximation of the Hodge $-\star$ Operator in Curved Elements, in Spectral and High Order Methods for Partial Differential Equations, vol. 76 of Lecture Notes in Computational Science and Engineering, Springer, 2010, pp. 283-291.

[93] A. Palha And M. Gerritsma, A mass, energy, enstrophy and vorticity conserving $(M E E V C)$ mimetic spectral element discretization for the $2 D$ 
incompressible Navier-Stokes equations, Journal of Computational Physics, 328 (2017), pp. 200-220.

[94] A. PALHA, B. Koren, AND F. Felici, A mimetic spectral element solver for the GradShafranov equation, Journal of Computational Physics, 316 (2016), pp. 63-93.

[95] A. Palha, P. P. Rebelo, and M. Gerritsma, Mimetic Spectral Element Advection, in Spectral and High Order Methods for Partial Differential Equations - ICOSAHOM 2012, vol. 95 of Lecture Notes in Computational Science and Engineering, Springer International Publishing, 2014, pp. 325-335.

[96] A. Palha, P. P. Rebelo, R. Hiemstra, J. Kreeft, and M. GerRITSMA, Physics-compatible discretization techniques on single and dual grids, with application to the Poisson equation of volume forms, Journal of Computational Physics, 257 (2014), pp. 1394-1422.

[97] D. Pavlov, P. Mullen, Y. Tong, E. Kanso, J. E. Marsden, And M. DESBRUN, Structure preserving discretization of incompressible fluids, Physica D: Nonlinear phenomena, 240 (2011), pp. 443-458.

[98] P. Perona AND J. MALIK, Scale-space and edge detection using anisotropic diffusion, IEEE Transactions on Pattern Analysis and Machine Intelligence, 12 (1990), pp. 629-639.

[99] J. B. Perot, Conservation properties of unstructured staggered mesh schemes, Journal of Computational Physics, 159 (2000), pp. 58-89.

[100] - Discrete conservation properties of unstructured mesh schemes, Annual Review of Fluid Mechanics, 43 (2011), pp. 299-318.

[101] J. B. Perot AND V. Subramanian, A discrete calculus analysis of the Keller Box scheme and a generalization of the method to arbitrary meshes, Journal of Computational Physics, 226 (2007), pp. 494-508.

[102] _ Discrete calculus methods for diffusion, Journal of Computational Physics, 224 (2007), pp. 59-81.

[103] J. B. Perot, D. Vidovic, AND P. Wesseling, Mimetic reconstruction of vectors, IMA Volumes in Mathematics and its Applications, 142 (2006), p. 173.

[104] F. RAPETTI, High order edge elements on simplicial meshes, ESAIM: Mathematical Modelling and Numerical Analysis, 41 (2007), pp. 1001-1020.

[105] — Whitney forms of higher order, SIAM J. Numer. Anal., 47 (2009), pp. 2369-2386.

[106] P. P. Rebelo, A. PAlha, AND M. Gerritsma, Mixed mimetic spectral element method applied to Darcy's problem, in Spectral and High Order Methods for Partial Differential Equations - ICOSAHOM 2012, vol. 95 of Lecture Notes in Computational Science and Engineering, Springer, 2014, pp. 373382.

[107] N. Robidoux, A New Method of Construction of Adjoint Gradients and Divergences on Logically Rectangular Smooth Grids, in Finite Volumes for Complex Applications: Problems and Persepctives, Éditions Hermès, Rouen, France, 1996, pp. 261-272. 
[108] __ Numerical solution of the steady diffusion equation with discontinuous coefficients, PhD thesis, University of New Mexico, Albuquerque, NM, USA, 2002.

[109] N. RobIDoux, Polynomial histopolation, superconvergent degrees of freedom, and pseudospectral discrete Hodge operators, Unpublished: http://people.math.sfu.ca/ nrobidou/public_html/prints/histogram/histogram.pdf, (2008).

[110] N. Robidoux AND S. Steinberg, A discrete vector calculus in tensor grids, Computational Methods in Applied Mathematics, 11 (2011), pp. 2366.

[111] M. SHASHKov, Conservative finite-difference methods on general grids, CRC Press, Boca Raton, FL, USA, 1996.

[112] C. Sovinec, A. Glasser, T. Gianakon, D. Barnes, R. Nebel, S. Kruger, D. Schnack, S. Plimpton, A. Tarditi, M. Chu, and $\mathrm{N}$. TEAM, Nonlinear magnetohydrodynamics simulation using high-order finite elements, Journal of Computational Physics, 195 (2004), pp. 355-386.

[113] S. SteInBERG, A discrete calculus with applications of higher-order discretizations to boundary-value problems, Computational Methods in Applied Mathematics, 42 (2004), pp. 228-261.

[114] S. Steinberg AND J. P. Zingano, Error estimates on arbitrary grids for 2nd-order mimetic discretization of Sturm-Liouville problems, Computational Methods in Applied Mathematics, 9 (2009), pp. 192-202.

[115] T. Tarhasani, L. Kettunen, And A. Bossavit, Some realizations of a discrete Hodge operator: a reinterpretation of finite element techniques, IEEE Transactions on Magnetics, 35 (1999).

[116] G. I. TAYLOR, Production and dissipation of vorticity in a turbulent fluid, Proceedings of the Royal Society A: Mathematical, Physical and Engineering Sciences, 164 (1938), pp. 15-23.

[117] E. TONTI, On the formal structure of physical theories, tech. rep., Italian National Research Council, 1975.

[118] Y. Wang, H. Hajibeygi, And H. A. TChelePI, Algebraic multiscale solver for flow in heterogeneous porous media, Journal of Computational Physics, 259 (2014), pp. 284-303.

[119] H. Whitney, Geometric integration theory, Dover Publications, Inc., 1957.

[120] X.-H. WU AND R. PARAShKeVOV, Effect of grid deviation on flow solutions, SPE Journal, 14 (2009), pp. 67-77.

[121] A. Younes, P. ACKERER, AND F. DELAY, Mixed finite elements for solving 2-D diffusion-type equations, Reviews of Geophysics, 48 (2010), p. RG1004.

[122] L. C. YounG, Rigorous treatment of distorted grids in 3D, in SPE Reservoir Simulation Symposium, Society of Petroleum Engineers, 1999.

[123] X. Zhang, Schmidt, D., AND J. B. Perot, Accuracy and conservation properties of a theree-dimensional unstructured staggered mesh scheme for fluid dynamics, Journal of Computational Physics, 175 (2002), pp. 764-791. 